\title{
Estimativas de entropia e um resultado de existência de ferraduras para uma teoria de forcing de homeomorfismos de superfícies
}

\author{
Everton Juliano da Silva
}

TESE APRESENTADA

AO

InstiTUTO DE MATEMÁTICA E EsTATÍSTICA

DA

UNIVERSIDADE DE SÃO PAULO

PARA

OBTENÇÃO DO TÍTULO

$\mathrm{DE}$

DOUTOR EM CIÊNCIAS

Programa: Doutorado em Matemática aplicada

Orientador: Prof. Dr. Fábio Armando Tal

Durante o desenvolvimento deste trabalho o autor recebeu auxílio financeiro da FAPESP, CNPq e CAPES.

São Paulo, julho de 2019 


\title{
Estimativas de entropia e um resultado de existência de ferraduras para uma teoria de forcing de homeomorfismos de superfícies
}

\begin{abstract}
Esta versão da Tese contém as correções e alterações sugeridas pela Comissão Julgadora durante a defesa da versão original do trabalho, realizada em 17/06/2019. Uma cópia da versão original está disponível no Instituto de Matemática e

Estatística da Universidade de São Paulo.
\end{abstract}

Comissão Julgadora:

- Prof. Dr. Fábio Armando Tal (Presidente) - IME-USP

- Prof. Dr. André Salles de Carvalho - IME - USP

- Prof. Dr. Alejandro Kocsard - UFF

- Prof. Dr. Philip Lewis Boyland - UF

- Prof. Dr. Alejandro Miguel Passeggi Diaz Robles - FEAUDELAR 


\section{Agradecimentos}

Agradeço primeiramente aos meus pais, Laurípedes e Moisés, que sempre estiveram me apoiando, mesmo não sabendo no que eu estava trabalhando. Pessoas simples, que apenas com a quarta série sempre lutaram para ter uma vida melhor e poder me dar todo o apoio que eu precisei em toda minha vida. Vida esta que nunca foi de luxo, mas o essencial sempre esteve presente. Por isto, meus sinceros agradecimentos.

Mariana Nani, me desculpe os momentos de estresse, principalmente na fase final da elaboração deste trabalho. Só tenho a lhe agradecer por todos os momentos juntos, ao carinho e amor e dedicação que sempre teve para comigo. Nunca esqueça o quanto eu amo você.

Ao meu orientador, Fábio Tal, meu muitíssimo obrigado. O acaso me colocou como seu orientando, e só tenho a lhe agradecer toda ajuda e suporte dado a mim. Obrigado também pela sugestão e oportunidade que me foi dada de estudar fora do país que foi uma das experiências mais incríveis que vivenciei. A jornada foi longa, mas sinto que passou rápido demais. Só lhe tenho gratidão e lhe desejo sucesso em seu trabalho e felicidades com sua família.

A special thanks goes to Philp Boyland from University of Florida. I am so grateful for the opportunity to study with you. Unfortulately, We didn't have to much time, but I could enjoy all the opportunities that we have. Thank you for gave me this amazing experience.

Another special thanks goes to Keith and Sharon from Gainesville. You helped me a lot when I was in this beautifull city. Both of you are amazing people and I just could study in University of Florida because of you. Thank you so much and go Gators.

Outra pessoa que não poderia ficar fora desta lista é minha amiga Pollyanna. Obrigado por me ouvir nos dias difíceis e por me dar apoio. Um muitíssimo obrigado por ler este texto e sugerir correções e gratidão por ouvir meus seminários. Talvez você não saiba, mas no fundo você mais me ajudou do que eu lhe ajudei.

Agradeço também aos meus amigos Bruno, Diego e Guilherme. Vivenciamos praticamente toda essa jornada juntos e lhes desejo todo o sucesso que a vida 
possa lhes proporcionar.

Muitas pessoas passam por nossas vidas e de certa forma, nos moldam e nos transformam no que somos hoje. Cito como exemplo nossos amigos e nossos professores, desde o primário até a universidade. Aqui vai meu muito obrigado para todos vocês, que me ajudaram em toda essa jornada. Gratidão especial para o professor Salvador Zanata, que me ajudou em minha qualificação como presidente da banca e ao professor Sylvain Bonnot, que me aceitou como aluno do programa PAE.

Gratidão para com toda minha família, que sempre torceu para meu sucesso. Um obrigado especial vai para meus familiares da Bahia, que mesmo estando tão distantes, sempre estiveram comigo guardados em meu peito.

Agradeço imensamente à FAPESP, CNPq e CAPES pelo apoio financeiro. 


\section{Resumo}

Neste trabalho estudamos o valor mínimo da entropia topológica para uma classe de aplicações isotópicas à identidade em superfícies orientáveis (sem bordo, não necessariamente compactas e possivelmente de tipo finito) sob um ponto de vista estritamente topológico. Este estudo é feito utilizando a nova teoria de forcing para trajetórias transversas de Le Calvez e Tal que se baseia na teoria de Brouwer equivariante, em que é possível folhear superfícies com folhas relacionadas a teoria de Brouwer no plano.

O principal resultado deste trabalho é uma melhora na estimativa da entropia topológica obtida por Le Calvez e Tal em um recente trabalho em que os autores buscam ferraduras topológicas em superfícies orientáveis utilizando ferramentas similares apresentadas aqui. Uma aplicação deste resultado acima é feita utilizando aplicações em $\mathbb{S}^{2}$ que possuam um ponto fixo cuja trajetória pela isotopia deste ponto não seja homotópica a um múltiplo de um loop simples. Com estas hipóteses, melhoramos a estimativa dada por Le Calvez e Tal em que é encontrado um valor mínimo estritamente positivo para a entropia topológica desta aplicação.

Palavras chaves: Teoria de forcing, entropia topológica, ferradura topológica, homeomorfismos em superfícies. 


\section{Abstract}

In this work we study the minimum topological entropy value for one class of maps isotopics to the identity in oriented surfaces (without border, not necessary compacts and possibly of finite type) under the point of view strictly topological. This study is done using the new forcing theory to transverse trajectories from Le Calvez and Tal which it is based to equivariant Brouwer Theory, on what it is possible to leaf surfaces with leaves related to plane Brouwer theory.

The main result in this work is a improvement in the estimates from the topological entropy obtained by Le Calvez and Tal in one recent work where the authors seek topological horseshoes on oriented surfaces using tools very similar to that are shown here. One application of the above result is done using maps on $\mathbb{S}^{2}$ that have a fixed point whose trajectory by the isotopy of this point do not be homotopic to a multiple of a simple loop. With these hypotheses, we improve the estimates given by Le Calvez and Tal on what is found a strictly positive minimum value to the topological entropy of this map.

Keywords: forcing theory, topological entropy, topological horseshoe, homeomorphisms on surfaces. 


\section{Sumário}

Agradecimentos

Resumo iii

Abstract

$\begin{array}{ll}\text { Introdução } & 1\end{array}$

1 Preliminares $\quad 8$

1.1 Entropia e deslocamento de Bernoulli . . . . . . . . . . . . 8

1.2 Ferradura topológica . . . . . . . . . . . . . . . . . . . . 9 9

2 Teoria de Brouwer, Teoria de Brouwer equivariante e isotopias maximais 12

2.1 Teoria de Brouwer . . . . . . . . . . . . . . . . 13

2.2 Isotopias Maximais . . . . . . . . . . . . . . . . . . . 15

2.3 Teoria de Brouwer equivariante . . . . . . . . . . . 16

$\begin{array}{llr}3 & \text { Forcing theory } & 20\end{array}$

4 Estimativa para a entropia $\quad 24$

4.1 Resultado principal . . . . . . . . . . . . . . . . . . . . 24

4.2 Aplicação . . . . . . . . . . . . . . . . . . 45

$\begin{array}{ll}\text { Referências } & 47\end{array}$ 


\section{Introdução}

Segundo Viana e Oliveira ([1]), a palavra entropia foi concebida pelo físico e matemático Rudolf Clausius e que na teoria dos sistemas dinâmicos em equilíbrio, a entropia é de certa forma uma medida do grau de desordem do sistema. Andrey Kolmogorov e Yakov Sinai, segundo Viana e Oliveira, proporam uma definição de entropia $h_{\mu}$ de um sistema em teoria ergódica utilizando medidas de probabilidade invariantes, cujo principal objetivo era fornecer um invariante de equivalência ergódica que pudesse distinguir dois deslocamentos de Bernoulli. A definição de entropia topológica $h$, que é a que usaremos neste trabalho, foi dada por Adler, Konhein e McAndrew ([2]) e é um invariante topológico para uma transformação contínua $f: M \rightarrow M$ em um espaço métrico compacto. Bowen ([3]) definiu entropia para o caso de $f$ ser uma transformação contínua e $M$ um espaço métrico (não necessariamente compacto) e é sabido que ambas definições são equivalentes no caso de $M$ ser um espaço métrico compacto. Um belo teorema de teoria ergódica chamado de princípio variacional faz a relação entre a entropia de Kolmogorov e Sinai e a entropia topológica e diz que esta última coincide com o supremo de $h_{\mu}$ tomado sobre todas as medidas de probabilidades invariantes $\mu$.

Um elegante resultado de Katok ([4]) diz que se $f$ é um $C^{1+\alpha}$ difeomorfismo, $\alpha>0$, em uma superfície compacta $S$ com entropia topológica estritamente positiva, então existe um conjunto compacto $\Lambda \subseteq S$ que é $f$ invariante tal que alguma potência de $\left.f\right|_{\Lambda}$ é conjugada ao shift bilateral. Segue então que com estas hipóteses, $f$ tem uma ferradura. Entretanto, essa relação entre entropia positiva em difeomorfismos suficientemente suaves e a existência de ferraduras não se estende aos homeomorfismos. Ress ([5]) construiu um homeomorfismo $f$ do toro $\mathbb{T}^{d}(d \geq 2)$ com entropia positiva mas que não possui nenhuma órbita periódica (de fato, o homeomosfismo contruído $f$ é minimal e portanto, sem pontos periódicos). Os pesquisadores Béguin, Crovisier e Le Roux estenderam o trabalho de Ress em ([6]), o que ressalta ainda mais a não aplicabilidade do resultado de Katok para homeomorfismos.

Novos resultados para homeomorfismos isotópicos à identidade vem apare- 
cendo com a recente teoria de forcing forcing para trajetórias transversas de homeomorfismos em superfícies desenvolvida por Tal e Le Calvez ([16]). Provavelmente o exemplo mais conhecido sobre forcing em sistemas dinâmicos seja o chamado teorema de Sharkovsky: Existe uma ordem total $\preceq$ sobre o conjunto dos inteiros estritamente positivos tal que dada uma transformação contínua $f:[0,1] \rightarrow[0,1]$ que contenha uma órbita periódica de período $m$, então para todo $n \preceq m, f$ contém uma órbita periódica de período $n$. A teoria de forcing para trajetórias transversas é baseada na teoria de Brouwer para homeomorfismos do plano que preservam a orientação sem pontos fixos, chamados de homeomorfismos de Brouwer e na existência de uma folheação contínua singular e orientável $\mathcal{F}$ para homeomorfismos isotópicos à identidade em superfícies, demonstrada por Le Calvez ([13]).

Dado $f$ um homeomorfismo isotópico à identidade, e se $\mathcal{I}$ denota o conjunto das itotopias da identidade para $f$, é possível definir uma ordem parcial em $\mathcal{I}$ da seguinte forma: $I \preceq I^{\prime}$ se

- $\operatorname{fix}(I) \subseteq \operatorname{fix}\left(I^{\prime}\right)$

- $I^{\prime}$ é homotópico a $I$ relativo à fix $(I)$.

Os pesquisadores Béguin, Crovisier e Le Roux ([18]) provam que para qualquer $I \in \mathcal{I}$, sempre existe isotopia $I^{\prime} \in \mathcal{I}$ com $I \preceq I^{\prime}$ e $I^{\prime}$ maximal. Com isso em mãos, dada $I$ isotopia em $\mathcal{I}$ denote fix $(I)$ o conjunto dos pontos fixos da isotopia. Se $I$ for maximal e se $\widetilde{f}$ denota o levantamento de $f$ dado pela isotopia no espaço de recobrimento universal de $\widetilde{\operatorname{dom}}(I)=M \backslash$ fix $(I)$, então $\widetilde{f}$ é um homeomorfismo de Brouwer em cada componente conexa de $\widetilde{\operatorname{dom}}(I)$ (que podemos supor, para o propósito deste trabalho, que seja conexo). Alem disso, usando o resultado de Le Calvez citado acima, existe uma folheação orientada contínua $\mathcal{F}$ em $M$ cujos pontos singulares coincidem com os pontos fixos da isotopia $I$. A folheação restrita ao conjunto não singular quando levantada para $\widetilde{\operatorname{dom}}(I)$, é uma folheação formada por linhas de Brouwer para $\tilde{f}$, denotada por $\widetilde{\mathcal{F}}$.

A teoria de forcing de Le Calvez e Tal nos dá novas órbitas para homeomorfismos de superfícies orientáveis isotópicas à identidade em termos de isotopias maximais, folheações, caminhos transversos e admissíveis e intersecções transversas. Através destas novas órbitas, Tal e Le Calvez ([19]) encontraram ferraduras 
topológicas em uma classe de homeomorfismos isotópicos à identidade que possuem uma trajetória com autointersecção transversa, um conceito a ser explicado melhor no corpo desta tese. Com isso em mãos, eles foram capazes de estimar a entropia topológica mínima para estas aplicações. Os pesquisadores aplicaram este resultado no estudo de homeomorfismos de superfícies de gênero zero que não possuem ferradura topológica. Várias aplicações deste estudo foram deduzidas, dentre elas, podemos citar a extensão do trabalho de Franks e Handel ([7]), que apresentaram um teorema de estrutura para difeomorfismos $C^{\infty}$ da esfera que preservam área, que Tal e Le Calvez estenderam para o caso $C^{0}$.

Teorema 0.1. Seja $f: \mathbb{S}^{2} \rightarrow \mathbb{S}^{2}$ um homeomorfismo que preserva orientação e que não possua ferradura topológica. Então o conjunto

$$
\Omega^{\prime}(f)=\{z \in \Omega(f): \alpha(z) \cup \omega(z) \nsubseteq \operatorname{fix}(f)\}
$$

podem ser cobertos por uma familia $\left(A_{\alpha}\right)_{\alpha \in \mathcal{A}}$ de subconjuntos invariantes tal que:

1. $A_{\alpha}$ é um anel topológico aberto livre de pontos fixos e $\left.f\right|_{A_{\alpha}}$ é isotópico à identidade.

2. Se $\kappa$ é um gerador de $H_{1}\left(A_{\alpha}, \mathbb{Z}\right)$, então existe um levantamento de $\left.f\right|_{A_{\alpha}}$ para o espaço de recobrimento universal de $A_{\alpha}$ cujos números de rotação estão todos inclusos em $[0,1]$.

3. $A_{\alpha}$ é maximal para as duas propriedades anteriores.

Outra aplicabilidade da teoria se traduz em vários resultados sobre a teoria dos números de rotação para homeomorfismos do anel que não possuem ferraduras topológicas. Denote $\mathbb{T}^{1}=\mathbb{R} / \mathbb{Z}$ e considere $\check{\pi}: \mathbb{R}^{2} \rightarrow \mathbb{A}$ a projeção de recobrimento do anel $\mathbb{A}=\mathbb{T}^{1} \times \mathbb{R}$. Seja $f$ um homeomorfismo isotópico à identidade em $\mathbb{A}$ e considere $\check{f}$ um levantamento para $\mathbb{R}^{2}$. É dito que um ponto $z \in \mathbb{A}$ é um ponto escaping para $f$ se a sequência $\left(f^{n}(z)\right)_{n \geq 0}$ converge para algum fim de $\mathbb{A}$. Denote por $\mathrm{ne}^{+}(f)$ o complemento do conjunto dos pontos escaping. Denote também ne $(f)=$ ne $^{+}\left(f^{-1}\right)$. Defina ne $(f)=$ ne $^{+}(f) \cup$ ne $^{-}(f)$ e seja $\Omega(f)$ o conjunto dos pontos não errantes. É dito que $z \in \mathrm{ne}^{+}(f)$ tem um número 


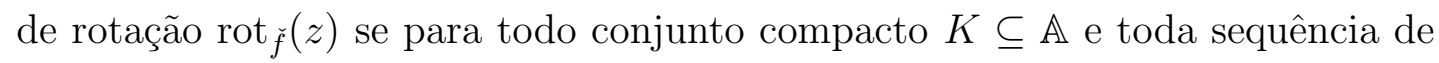
inteiros crecentes $\left(n_{k}\right)_{k \geq 0} \operatorname{com} f^{n_{k}}(z) \in K$, temos

$$
\lim _{k \rightarrow+\infty} \frac{1}{n_{k}}\left(\pi_{1}\left(\check{f}^{n_{k}}(\check{z})-\pi_{1}(\check{z})\right)\right)=\operatorname{rot}_{\check{f}}(z)
$$

se $\check{z}$ for um levantamento de $z$ e $\pi_{1}$ a projeção na primeira coordenada.

Teorema 0.2. Seja $f$ um homeomorfismo de $\mathbb{A}$ isotópico à identidade e $\breve{f}$ um levantamento de $f$ para $\mathbb{R}^{2}$. Suponha que $f$ não tenha ferradura topológica. Então

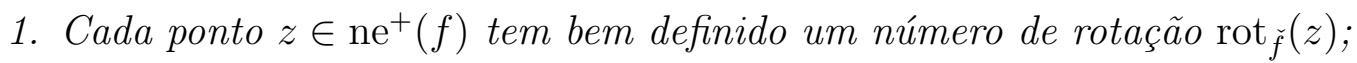

2. Para todos os pontos $z, z^{\prime} \in \mathrm{ne}^{+}(f)$ tal que $z^{\prime} \in \omega(z)$, temos $\operatorname{rot}_{\tilde{f}}\left(z^{\prime}\right)=$ $\operatorname{rot}_{\check{f}}(z)$.

3. Se $z \in \mathrm{ne}^{+}(f) \cap \mathrm{ne}^{-}(f)$ é ñ̃o errante, então $\operatorname{rot}_{\breve{f}^{-1}}(z)=-\operatorname{rot}_{\tilde{f}}(z)$.

4. A aplicação $\operatorname{rot}_{\check{f}}: \Omega(f) \cap \mathrm{ne}(f) \rightarrow \mathbb{R}$ é contínua, onde

$$
\operatorname{rot}_{\breve{f}}(z)=\left\{\begin{array}{cc}
\operatorname{rot}_{\check{f}}(z) & \text { se } z \in \Omega(f) \cap \mathrm{ne}^{+}(f), \\
-\operatorname{rot}_{\check{f}^{-}}(z) & \text { se } z \in \Omega(f) \cap \mathrm{ne}^{-}(f) .
\end{array}\right.
$$

Para mais detalhes deste resultado e outros resultados referentes ao conjunto de rotação do anel para homeomorfirmos que não possuam ferradura topológica, veja [19].

Outro tópico bastante interessante abordado em [19] é o estudo de homeomorfismos dissipativos do plano com entropia zero com o propósito de descrever a dinâmica dos difeomorfismos que podem ser aproximadas por aplicações com entropia estritamente positiva ([20], [21], [22]).

Um conjunto compacto invariante $X$ é localmente estável se $X$ admite um sistema fundamental de vizinhanças invariantes para frente. Uma aplicação $f: \mathbb{S}^{2} \rightarrow \mathbb{S}^{2}$ é topologicamente infinitamente renormalizável sobre um conjunto invariante fechado não vazio $\Lambda$ se existe uma sequência crescente $\left(q_{n}\right)_{n \geq 0}$ de inteiros positivos e uma sequência $\left(D_{n}\right)_{n \geq 0}$ de discos abertos tal que:

- $q_{n}$ divide $q_{n+1}$; 
- $D_{n}$ é $f^{q_{n}}$ periódico;

- os discos $f^{k}\left(D_{n}\right), 0 \leq k<q_{n}$ são dois a dois disjuntos;

- $\Lambda \subseteq \cup_{0 \leq k<q_{n}} f^{k}\left(D_{n}\right)$.

Assim, temos o próximo resultado ([19]):

Proposição 0.3. Seja $f: \mathbb{R}^{2} \rightarrow \mathbb{R}^{2}$ um difeomorfismo que preserva orientação que não possua ferradura, tal que $|\operatorname{det} D f(x)|<1$ para todo $x \in \mathbb{R}^{2}$. Seja $\Lambda$ um conjunto compacto transitivo que é localmente estável. Então ou $\Lambda$ é uma órbita periódica ou $f$ é topologicamente infinitamente renormalizável sobre $\Lambda$.

Como pode-se perceber, toda essa gama de resultados (citados acima ou outros que podem ser encontrados em [19]), só foram possíveis graças a teoria de forcing para trajetórias transversas e principalmente, a descoberta de ferraduras para uma classe importante de homeomorfismos isotópicos à identidade. Nesta tese, os autores melhoram o principal resultado de [19] encontrando novas ferraduras, o que possibilitou melhores estimativas da entropia topológica para a mesma classe de homeomorfismos de superfícies isotópicas à identidade estudades por Le Calvez e Tal. Esta classe de homeomorfismos compreende os homeomorfismos que possuam um caminho $n$-admissível com autointersecção transversa (noções estas que serão definida nos capítulos seguintes). De fato, mostraremos o seguinte teorema:

Teorema 0.4. Seja $M$ uma superfície orientada, $f$ um homeomorfismo de $M$ isotópico à identidade, I uma isotopia maximal de $f$ e $\mathcal{F}$ uma folheação transversa para $I$. Se $\gamma:[a, b] \rightarrow \operatorname{dom}(I)$ é um caminho admissivel de ordem $n e$ se $\gamma$ tem uma autointersecção transversa, então a entropia de $f$ é pelo menos $\log (2) / n$.

Embora os autores deste presente texto encontraram novas ferraduras e melhoraram a estimativa mínima da entropia topológica, uma pergunta ainda contínua em aberto: 
Pergunta: A melhor estivativa mínima de entropia topológica para o Teorema 0.4 é $\log (2) / n$ ?

Em outras palavras, existe alguma aplicação isotópica à identidade que possua um caminho $n$-admissível transverso para a folheação $\mathcal{F}$ com uma autointersecção transversa com entropia exatamente $\log (2) / n$ ?

Outro resultado provado neste trabalho, que é uma aplicação praticamente direta de 0.4, é o seguinte:

Teorema 0.5. Seja $f$ um homeomorfismo que preserva a orientação em $\mathbb{S}^{2} e$ I uma isotopia maximal. Assuma que existe um ponto $x \in \operatorname{dom}(I)$ fixo para $f$ tal que o loop naturalmente definido pela trajetória $I(x)$ não é homotópico em $\operatorname{dom}(I)$ a um múltiplo de um loop simples. Então a entropia de $f$ é pelo menos igual a $\log (2) / 2$.

Uma versão do teorema acima foi primeiramente demonstrado por Le Calvez e Tal ([16]), que provaram que $f$ deve ter entropia mínima de $\log (2) / 4$. Aqui, graças ao 0.4, foi possível melhorar estimativa da entropia topológica de $f$.

Este trabalho está estruturado da seguinte forma:

No capítulo 1 é exibido as propriedades básicas relativas a entropia topológica que foi utilizada neste texto e sobre o shift (ou deslocamento) de Bernoulli. Além disso, é definido ferradura topológica e é feito um resumo do trabalho de Kennedy e Yorke sobre a existência destas para uma classe de aplicações que possuam a chamada hipóteses de ferradura $\Omega$. O principal resultado de Kennedy e Yorke é uma ferramenta chave para o resultado principal desta tese.

No capítulo 2 temos o ferramental básico para o entendimento da teoria de forcing para homeomorfirmos em superfícies orientáveis. Situaremos o leitor às terminologias básicas como linha de Brouwer, isotopia maximal e caminhos transversos. Neste capítulo é enunciado o principal teorema de [13] que fala sobre a existência de folheações não singulares do plano formada de linhas de Brouwer para um homeomorfismo de Brouwer $f$, e também a existência de folheações singulares em superfícies para um homeomorfirmo $f$ com propriedades análogas.

No capítulo 3 temos a noção de classe de equivalência para caminhos transversos, a definição de caminhos $n$-admissíveis e a de caminhos com intersecção 
transversa (em particular, a de caminhos com autointersecção transversa), e por fim, a teoria de forcing para homeomorfismos em superfícies orientáveis de Le Calvez e Tal ([16]).

No capítulo 4 é apresentado é feio a prova do principal resultado deste trabalho. É construído um conjunto $Q$ contido em $M$ que possui propriedades semelhantes a uma ferradura topológica, mas que não é compacto. Com uma compactificação apropriada e utilizando aproximações, o resultado é provado. Ainda neste capítulo o Teorema 0.5 é demonstrado. 


\section{Entropia para trajetórias transversas}

\section{Preliminares}

\subsection{Entropia e deslocamento de Bernoulli}

Neste trabalho vamos pressupor que o leitor já tenha tido contato prévio com as definições de entropia topológica e seus principais resultados. Uma boa referência para este conceito pode ser encontrado no livro do Viana e Oliveira ([1]). Neste trabalho, definiremos a entropia de uma aplicação contínua $f: M \rightarrow M \mathrm{em}$ uma superfície orientável $M$, que denotaremos por $h(f)$, como sendo a entropia topológica de sua extensão para a compactificação de Alexandrov de $M$ que fixa o ponto no infinito.

No que segue, elencaremos algumas propriedades da entropia cujas provas podem ser encontradas em ([1]). Se $A \subseteq B \subseteq M$ são compactos invariantes por $f$, então $h\left(\left.f\right|_{A}\right) \leq h\left(\left.f\right|_{B}\right)$. Caso $f$ seja um homeomorfismo, então $h\left(f^{z}\right)=|z| h(f)$, para todo $z$ inteiro. Sejam $f: M \rightarrow M$ e $g: N \rightarrow N$ aplicações contínuas em dois espaços métricos compactos $M$ e $N$. Dada uma aplicação contínua e sobrejetora $u: M \rightarrow N$, dizemos que $u$ é uma semiconjugação entre $f$ e $g$ se $u \circ f=g \circ u$. Neste caso, $h(f) \geq h(g)$ e vale a igualdade caso $u$ seja um homeomorfismo. No caso de $u$ não ser homeomorfismo, a igualdade não necessariamente é válida, entretanto, se existir algum $K>0$ tal que $\left|u^{-1}(x)\right| \leq K, \forall x \in M$, vale a igualdade.

Uma transformação contínua $f: M \rightarrow M$ em um espaço métrico compacto é chamada de expansiva se existe $\delta>0$ tal que $d\left(f^{i}(x), f^{i}(y)\right)<\delta$ para todo $i \in \mathbb{N}$ implica que $x=y$. Denote por $\operatorname{Fix}\left(f^{n}\right)$ o conjunto dos pontos $x$ em $M$ tais que $f^{n}(x)=x$. Então temos a seguinte proposição:

Proposição 1.1. Se $M$ é um espaço métrico compacto e $f: M \rightarrow M$ expansiva então

$$
\limsup _{n} \frac{1}{n} \log \# \operatorname{Fix}\left(f^{n}\right) \leq h(f) .
$$


Uma demosntração da proposição acima pode ser encontrada em [1]. Considere $\Sigma_{n}=\{0, \ldots, n-1\}^{\mathbb{Z}}$ o conjunto das sequências bilaterais indexadas em $\mathbb{Z}$ com entradas $0, \ldots, n-1$. Considere a dinâmica $\sigma: \Sigma_{n} \rightarrow \Sigma_{n}$ dada por $\sigma\left(\left(x_{k}\right)_{k \in \mathbb{Z}}\right)=\left(x_{k+1}\right)_{k \in \mathbb{Z}}$, ou seja, a sequência é deslocada 'para trás' uma vez. Essa dinâmica é conhecida como deslocamento (ou shift bilateral) de Bernoulli em $n$ símbolos. É possível colocar uma métrica nesse espaço da seguinte forma: dados $x=\left(x_{k}\right)_{k \in \mathbb{Z}}$ e $y=\left(y_{k}\right)_{k \in \mathbb{Z}}, d(x, y)=2^{-|r|}$, onde $|r|$ é o primeiro natural tal que a coordenada $x_{r}$ e $y_{r}$ são diferentes, e $d(x, y)=0$ caso $x=y$. Nesta métrica $\sigma$ é um homeomorfismo e é possível mostrar (veja [1]) que a transformação $\sigma$ é expansiva e que $h(\sigma)=\log (n)$. Analogamente, é possível construir a dinâmica em $\Sigma_{n}^{+}=\{0, \ldots, n-1\}^{\mathbb{N}}$. Este último é conhecido como o espaço das sequências unilaterais, ou shift unilateral em $n$ símbolos. Neste caso $\sigma$ (definido de forma análoga) continua sendo uma transformação contínua, com entropia $h(\sigma)=\log (n)$ mas não mais um homeomorfismo.

\subsection{Ferradura topológica}

A primeira aparição do que chamamos de ferradura foi dada por Stephen Smale ([8]) e basicamente consiste de um difeomorfismo $f$ do plano no qual existe um conjunto $Q$ (difeomorfo a um retângulo) que é linearmente contraído horizontalmente, esticado linearmente verticalmente e depois dobrado de forma que fique parecendo uma ferradura de cavalo e que quando intersectado com Q, obtém-se dois retângulos disjuntos (Veja figura 1). Nesta situação, Smale mostrou que existe um subconjunto $\Lambda$ de $Q$, compacto, invariante por $f$ tal que $\left.f\right|_{\Lambda}$ é topologicamente conjugado à aplicação shift com dois símbolos.

Definição 1.2. Dado um homeomorfismo $f: M \rightarrow M$ em uma superfície orientável $M$, dizemos que um homeomorfismo $g: Z \rightarrow Z$ definido sobre espaço hausdorff compacto é uma extensão de $f$ caso exista uma transformação contínua e sobrejetora $H: Z \rightarrow M$ tal que $H \circ g=f \circ H$. Além disso, dizemos que a extensão é finita, ou simplesmente extensão finita, caso as fibras da aplicação fator $h$ sejam todas finitas com uma limitação uniforme em sua cardinalidade, ou seja, que exista $A>0$ tal que $\# h^{-1}(x) \leq A, \forall x \in M$.

Tal e Le Calvez ([19]) definem ferradura topológica da seguinte forma: Se $X$ é 


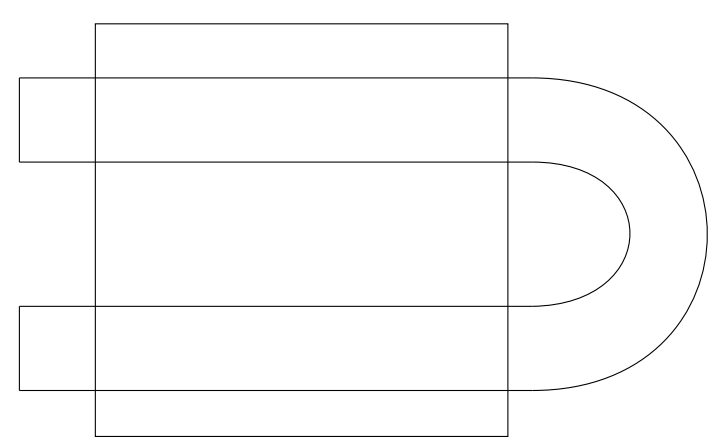

Figura 1: Ferradura de Smale.

um espaço topológico Hausdorff localmente compacto, um subconjunto compacto $Y \subseteq X$ é dito uma ferradura topológica para um homeomorfismo $f$ se é invariante por uma potência $f^{r}$ de $f$ e se $f^{r} \mid Y$ admite uma extensão finita $g: Z \rightarrow Z$ tal que:

- $g$ é uma extensão do shift bilateral de Bernoulli em $q \geq 2$ símbolos;

- A preimagem de toda sequência $s$ periódica de $\{0, \ldots, q-1\}^{\mathbb{Z}}$ pela aplicação fator $u: \mathbb{Z} \rightarrow\{0, \ldots, q-1\}^{\mathbb{Z}}$ contém um $s$ periódico ponto de $Z$.

Se $f: M \rightarrow M$ um homeomorfismo em uma superfície $M$, Burns e Weiss ([9]) dizem que $f$ tem uma ferradura topológica (ou ferradura geométrica) se existir um subconjunto $Q$ de $M$ compacto e invariante e também uma função contínua sobrejetora $u: Q \rightarrow \Sigma_{n}$ tal que $u \circ f=\sigma \circ u$ para algum $n \geq 2$, ou seja, que $\Sigma_{n}$ seja um fator de $f$. Neste trabalho, a menos de menção em contrário, estaremos pensando em ferraduras topológicas no sentido de Burns e Weiss. Kennedy e Yorke ([10]) mostraram a existência de ferraduras topológicas para uma classe de funções que possuam as hipóteses de ferradura $\Omega$ que descreveremos a seguir. Cada símbolo a seguir define uma hipótese:

- $\Omega_{X}$ : X é um espaço métrico separável.

- $\Omega_{Q}: Q \subseteq X$ é localmente conexo e compacto.

- $\Omega_{f}:$ A aplicação $f: Q \rightarrow X$ é contínua.

- $\Omega_{E}$ : Existem conjuntos end $d_{0}$ e $e n d_{1}$ contidos em $Q$, disjuntos e compactos e cada componente de $Q$ intersecta ambos $e n d_{0}$ e $e n d_{1}$. 
Uma conexão $\Gamma$ é um subconjunto de $Q$, compacto e conexo que intersecta ambos $e n d_{0}$ e $e n d_{1}$. É importante lembrar que um espaço métrico conexo e compacto também é chamado de contínuo. Neste trabalho, assim como em [10], permitiremos que um contínuo consista apenas de um ponto ou que seja o conjunto vazio. Um subconjunto de um contínuo que é um contínuo será chamado de subcontínuo. Um caminho em $Q$ que intersecta ambos end $d_{0}$ e $e n d_{1}$ é um exemplo de conexão. Uma preconexão $\gamma$ é um subconjunto compacto e conexo de $Q$ para o qual $f(\gamma)$ é uma conexão. Desta forma, Kennedy e Yorke definiram o crossing number $M$ (ou número de cruzamento $M$ ) como o maior número tal que toda conexão contém pelo menos $M$ preconexões mutualmente disjuntas. Assim, temos mais uma hipótese:

- $\Omega_{M}: Q$ tem crossing number $M \geq 2$.

As hipóteses $\Omega_{X}, \Omega_{Q}, \Omega_{f}, \Omega_{E}$ e $\Omega_{M}$ são chamadas coletivamente de hipóteses de ferradura $\Omega$. A figura 2, o exemplo padrão de Smale, mostra quem end 0 $e n d_{1}$ são e qualquer conexão que une $e n d_{0}$ e $e n d_{1}$ tem que possuir ao menos duas preconexões.

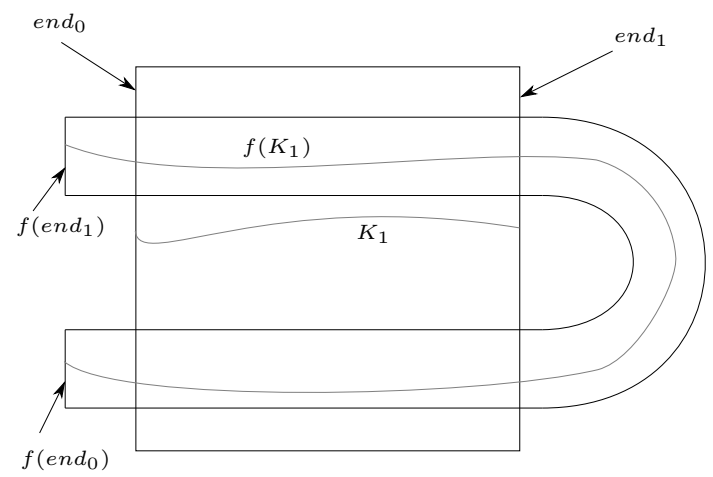

Figura 2: $K_{1}$ é uma conexão e possui 2 preconexões. Qualquer outra conexão terá ao menos duas preconexões, e portanto, $M=2$.

A figura 3 é um exemplo não trivial em que o número de cruzamentos $M$ vale 4. Um fato interessante sobre este exemplo é que ele foi obtido por um homeomorfismo isotópico à identidade em uma superfície orientável com uma folheação de Brouwer-Le Calvez que possui um caminho transverso a folheação com duas autointersecções transversas em relação a uma deck $T$. Veremos todas estas nomenclaturas e o que elas significam nos capítulos seguintes. 


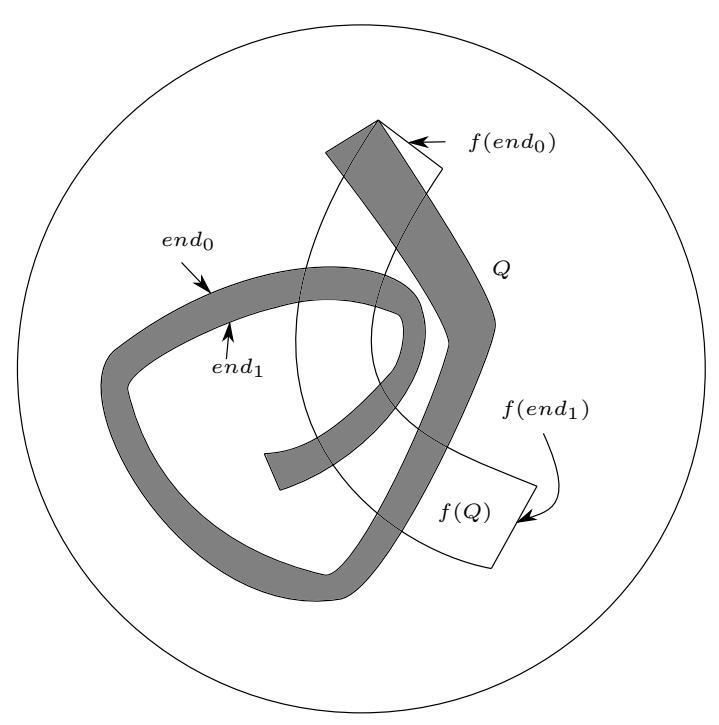

Figura 3: Qualquer conexão terá ao menos 4 preconexões. Neste caso, o número de cruzamentos $M$ é igual a 4.

Um subconjunto $S$ é invariante se $f(S)=S$. Assim, o principal teorema de [10] é o seguinte:

Teorema 1.3. Assuma as hipóteses de ferradura $\Omega$. Então existe um fechado invariante $Q_{I} \subseteq Q$ para o qual $\left.f\right|_{Q_{I}}$ é semiconjugada ao shift unilateral em $M$ símbolos. Se $f$ é um homeomorfismo, então $\left.f\right|_{Q_{I}}$ é também semiconjugada ao shift bilateral em $M$ símbolos.

Em outras palavas, quando se tem as hipóteses de ferradura $\Omega$ então $f$ tem uma ferradura topológica. Usaremos este teorema mais adiante.

\section{Teoria de Brouwer, Teoria de Brouwer equi- variante e isotopias maximais}

Neste capítulo introduzimos todo o ferramental básico para o entendimento da teoria de forcing para trajetórias transversas. É importante ressaltar que o conteúdo exposto nesse capítulo não é denso na teoria, o que significa que definições e resultados importantes não serão tratados aqui. A razão disto é para 
que este trabalho não fique demasiadamente extenso e focar no que é imprescindível para a teoria de forcing. A base se divide em três principais partes: teoria de Brouwer, isotopias maximais e teoria de Brouwer equivariante. No que segue, veremos cada uma destas três.

\subsection{Teoria de Brouwer}

Um homeomorfismo do plano que preserva a orientação e sem pontos fixos é chamado um homeomorfismo de Brouwer. Uma grande gama de exemplos de homeomorfismos de Brouwer vem dos fluxos no plano de tempo 1 e sem pontos fixos. Dizemos que uma aplicação $l: \mathbb{R} \rightarrow \mathbb{R}^{2}$ contínua é própria se a imagem inversa de qualquer subconjunto compacto de $\mathbb{R}^{2}$ é compacto de $\mathbb{R}$. Uma aplicação $l: \mathbb{R} \rightarrow \mathbb{R}^{2}$ contínua, injetiva e própria é chamada de linha. Neste trabalho, faremos um abuso de notação chamando também a imagem de $l$ de linha.

Um dos objetos de estudo mais importantes deste trabalho é a linha de Brouwer, que definiremos em breve. Primeiro, precisamos dar sentido no que seria a direita e a esquerda de uma linha, e é o que faremos a seguir. Dada uma curva $\alpha$ fechada e simples em $S^{2}$, a superfície esférica, podemos dar uma orientação para $\alpha$ induzida por uma parametrização, e assim podemos falar em direita $(D(\alpha))$ e esquerda $(E(\alpha))$ de $\alpha$. Assim temos o seguinte teorema:

Teorema 2.1 (Jordan-Schöenflies). Dados $\alpha:[0,1] \rightarrow S^{2}, \beta:[0,1] \rightarrow S^{2}$ curvas fechadas e simples. Então existe homeomorfismo $g: S^{2} \rightarrow S^{2}$ tal que $g(E(\alpha))=E(\beta), g(D(\alpha))=D(\beta)$ e $g([\alpha])=[\beta]$, onde $[\alpha]$ é a imagem de $\alpha$.

Uma consequência direta do teorema anterior é que se $l: \mathbb{R} \rightarrow \mathbb{R}^{2}$ uma linha, então existe homeomorfismo que preserva a orientação $g: \mathbb{R}^{2} \rightarrow \mathbb{R}^{2}$ tal que $g(l(t))=(0, t), \forall t \in \mathbb{R}$. Assim, definimos a direita de $l$ como sendo $R(l)=$ $g^{-1}((0,+\infty) \times \mathbb{R})$ e $\bar{R}(l)=g^{-1}([0,+\infty) \times \mathbb{R})$ o seu fecho. De forma análoga definimos a esquerda de $l$ e o seu fecho denotados por $L(l)$ e $\bar{L}(l)$ respectivamente.

Uma linha de Brouwer para um homeomorfismo de Brouwer no plano $f$ é uma linha $l$ tal que $f(l) \subseteq L(l)$ e $f^{-1}(l) \subseteq R(l)$ (Veja figura(4)). Em alguns textos, a definição de linha de Brouwer é a orientação contrária a esta que definimos. Vale ressaltar que não importa qual seja a escolha, ambas resultarão em teorias equivalentes. 


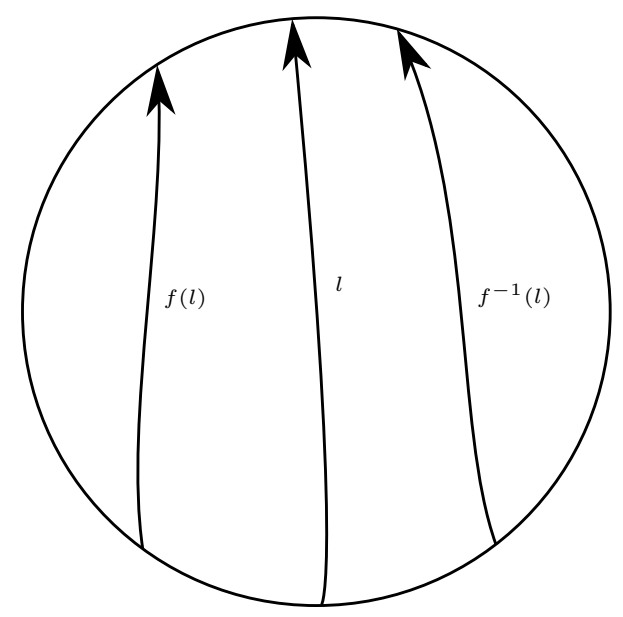

Figura 4: $l$ é uma linha de Brouwer para $f$.

Se $l$ é uma linha de Brouwer para $f$, é fácil ver que $f^{-1}(\bar{R}(l)) \subseteq R(l)$ e $f(\bar{L}(l)) \subseteq L(l)$. O principal resultado dessa seção é:

Teorema 2.2 (Teorema da translação de Brouwer). Se f é um homeomorfismo de Brouwer e $x$ um ponto do plano, então existe uma linha de Brouwer para $f$ que contém $x$.

Uma demonstração desse resultado pode ser encontrada em [11]. Como consequência, temos o seguinte corolário:

Corolário 2.3. Dados $f: \mathbb{R}^{2} \rightarrow \mathbb{R}^{2}$ homeomorfismo de Brouwer e $x \in \mathbb{R}^{2}$, existe $U \subset \mathbb{R}^{2}$ aberto simplesmente conexo, com $x \in U$, tal que $\left.f\right|_{U}: U \rightarrow U$ é conjugada ao homeomorfismo do plano $g(x)=x+(1,0)$. Além disso, todo ponto é errante, ou seja, $\Omega(f)=\emptyset$, onde $\Omega(f)$ é o conjunto dos pontos não-errantes de $f$.

O resultado acima nos diz que de uma certa forma, entendemos relativamente bem o comportamento dos homeomorfismos de Brouwer, pois são "localmente" conjugados a translação. O resultado abaixo é conhecido como lema dos discos livres, e será útil neste trabalho.

Lema 2.4. Seja $f: \mathbb{R}^{2} \rightarrow \mathbb{R}^{2}$ um homeomorfismo de Brouwer e seja $A$ um disco topológico do plano. Se $f(A) \cap A=\emptyset$ então $f^{k}(A) \cap A=\emptyset$ para todo $k \neq 0$ inteiro. 
Uma pergunta surge naturalmente do teorema da translação de Brouwer: Dado um homeomorfismo de Brouwer, é possível folhear o plano com linhas de Brouwer? Em 2004, Le Calvez deu a resposta afirmativa ([12]) a essa questão. Em 2005, Le Calvez ([13]) publicou uma versão equivariante do teorema de Brouwer, que veremos com mais detalhes adiante.

\subsection{Isotopias Maximais}

Uma homotopia $I$ entre dois homeomorfismos $f: M \rightarrow M$ e $g: M \rightarrow M$ definidos em uma superfície $M$ é uma função contínua $I:[0,1] \times M \rightarrow M$ tal que $I(0, x)=f(x)$ e $I(1, x)=g(x)$. Caso aconteça de para todo $t \in[0,1]$ a aplicação $I_{t}(x)=I(t, x)$ ser um homeomorfismo, diremos que $I$ é uma isotopia. Se $g$ é a aplicação identidade, diremos que $f$ é homotópica à identidade caso $I$ seja uma homotopia e que $f$ é isotópica à identidade caso $I$ seja uma isotopia. É importante observar que Epstein ([15]) mostrou que em uma superfície orientável fechada, dois homeomorfismos são homotópicos se e somente se são isotópicos.

Seja $f$ um homeomorfismo de uma superfície orientável $M$ isotópico à identidade e seja $I:[0,1] \times M \rightarrow M$ uma isotopia. O conjuntos dos pontos fixos de $I$, que é denotado por fix $(I)$, é o conjunto dos pontos que é fixado pela isotopia em todo o tempo, ou seja, $\operatorname{fix}(I)=\{x \in M: I(t, x)=x, \forall t \in[0,1]\}$. Se fix $(f)$ denota os pontos fixos de $f$, é claro que fix $(I) \subseteq \operatorname{fix}(f)$. O complementar de fix $(I)$ em $M$, chamado de domínio de $I$, será denotado por dom $(I)$.

Para cada ponto $x \in M$, a trajetória $I(x)$ de $x$ dado pela isotopia $I$ é dada por $I(x)(t)=I(t, x)$ para todo $t \in[0,1]$. Por concatenação, podemos construir para $n \geq 1$ a n-ésima trajetória de $x, I^{n}(x):[0, n] \rightarrow \operatorname{dom}(I)$, definido da seguinte forma: para cada $t \in[0, n]$, se $\lfloor t\rfloor$ é o maior inteiro menor ou igual a $t$, então $I^{n}(x)(t)=I\left(t-\lfloor t\rfloor, f^{\lfloor t\rfloor}(x)\right)$. Da mesma maneira, podemos definir a trajetória completa de $x$, denotada por $I^{\mathbb{Z}}(x)$ : se $t \in \mathbb{R}$, então $I^{\mathbb{Z}}(x)(t)=I\left(t-\lfloor t\rfloor, f^{\lfloor t\rfloor}(x)\right)$.

Dizemos que $I$ é maximal se não existe $x \in \operatorname{fix}(f) \backslash \operatorname{fix}(I)$ tal que o caminho da isotopia de $x$ seja homotópico a zero em $\operatorname{dom}(I)$. A ideia de maximal vem do fato que podemos colocar uma preordem nas isotopias da identidade de $f$ da seguinte forma: Dizemos que $I \preceq I^{\prime}$ se

- $\operatorname{fix}(I) \subseteq \operatorname{fix}\left(I^{\prime}\right)$ 
- $I^{\prime}$ é homotópico a $I$ relativo à fix $(I)$.

I é maximal se e somente se é maximal para a preordem acima (Veja [17]). Béguin, Crovisier e Le Roux (Veja [18]) provaram a existência das isotopias maximais:

Teorema 2.5. Para toda $I$ isotopia da identidade de $f: M \rightarrow M$, existe $I^{\prime}$ isotopia da identidade de $f$ tal que $I \preceq I^{\prime}$ e $I^{\prime}$ é maximal.

Se $I$ é isotopia maximal e se $\widetilde{I}$ denota a isotopia levantada de $\left.I\right|_{\operatorname{dom}(I)}$ em $\widetilde{\operatorname{dom}}(I)$, o espaço de recobrimento de $\operatorname{dom}(I)$, então o levantamento $\left.\widetilde{f} \operatorname{de} f\right|_{\operatorname{dom}(I)}$ para o espaço de recobrimento universal $\widetilde{\operatorname{dom}}(I)$ dado pela isotopia $\widetilde{f}(\cdot)=\widetilde{I}(1, \cdot)$ é livre de pontos fixos. Caso $\widetilde{\operatorname{dom}}(I)$ seja conexo, então é um plano topológico e portanto, $\widetilde{f}$ é um homeomorfismo de Brouwer. É importante notar que cada componente conexa de $\widetilde{\operatorname{dom}}(I)$ é um plano topológico. Neste trabalho, a menos de menção em contrário, vamos supor que $\widetilde{\operatorname{dom}}(I)$ é um plano topológico. O motivo disso é que pretendemos estudar caminhos transversos a folheação de Brouwer-Le Calvez (que será visto na próxima seção) e isso facilitará a notação, evitando que a todo momento tenhamos que dizer "a componente conexa de $\widetilde{\operatorname{dom}}(I)$ que contenha...".

\subsection{Teoria de Brouwer equivariante}

Nessa seção vamos enunciar o teorema de Brouwer equivariante, demonstrado por Le Calvez ([13]). Esse teorema é uma importante ferramenta, pois a partir dele, é possível estudar homeomorfismos isotópicos à identidade em superfícies utilizando folheações. Além disso, utilizando isotopias maximais, é possível associar esse homeomorfismo isotópico à identidade a um homeomorfismo de Brouwer no plano. Como os homeomorfismos de Brouwer no plano são relativamente mais fáceis de se estudar, é possível extrair resultados muito interessantes sobre os homeomorfismos isotópicos à identidade em superfícies. Vamos começar com uma definição:

Definição 2.6. Seja $G$ um grupo discreto de homeomorfismos do plano que preservam a orientação. Dizemos que $G$ age livremente $\mathrm{em} \mathbb{R}^{2}$ se $g x=x$ para algum 
$x$ no plano, implica que $g=I d$. Dizemos que $G$ age propriamente em $\mathbb{R}^{2}$ se para todo subconjunto compacto $K \subseteq \mathbb{R}^{2}$, temos que $g K \cap K \neq \emptyset$ para somente um número finito de $g \in G$.

Agora podemos enunciar o teorema equivariante de Brouwer:

Teorema $2.7([13])$. Sejam $f: \mathbb{R}^{2} \rightarrow \mathbb{R}^{2}$ um homeomorfismo de Brouwer e $G$ um grupo discreto de homeomorfismos do plano que preservam orientação, que age livremente e propriamente em $\mathbb{R}^{2}$. Se $f$ comuta com os elementos de $G$, então existe $\mathcal{F}$ folheação $G$-invariante de $\mathbb{R}^{2}$ por linhas de Brouwer de $f$.

Considere $M$ uma superfície e $\tilde{M}$ o seu espaço de recobrimento universal e considere $\pi$ a aplicação projeção. Uma deck de $\tilde{M}$ é um homeomorfismo $T$ tal que $\pi(\tilde{x})=\pi(T(\tilde{x})), \forall \tilde{x} \in \tilde{M}$. Uma deck também é chamada de automorfismo ou transformação de recobrimento. Denotaremos por $\operatorname{Deck}(M)$ o conjunto das decks de $M$. Note que $\operatorname{Deck}(M)$ é um grupo discreto de homeomorfismos do plano que preservam a orientação. Além disso, $\operatorname{Deck}(M)$ age livremente e propriamente em $\mathbb{R}^{2}$. Agora, se $f$ é um homeomorfismo isotópico à identidade em um superfície $M$, e se $\tilde{f}$ e $\tilde{M}$ são os respectivos levantamentos para o espaço de recobrimento universal, sabemos que nem sempre $\tilde{f}$ é livre de pontos fixos. Mas ao se utilizar isotopias maximais, podemos levantar $f$ para o espaço de recobrimento de $\operatorname{dom}(I)$ e, neste caso, o levantamento de $f$ dado pela isotopia levantada é livre de pontos fixos. Agora, se $\tilde{f}$ comutar com as transformações decks, então podemos aplicar o teorema de Brouwer equivariante, entretanto, da mesma forma que antes, não necessariamente $\tilde{f}$ comuta com as transformações decks.

Considere então $G=\operatorname{Deck}(M)$, e $I=[0,1] \times M \rightarrow M$ uma isotopia da identidade para $f$ e seja $\widetilde{I}=[0,1] \times \widetilde{M} \rightarrow \widetilde{M}$ a isotopia levantada para o espaço de recobrimento $\widetilde{M}$ de $M$. Se $\pi: \widetilde{M} \rightarrow M$ é a aplicação de projeção, então para toda $T \in G$, segue que $\pi(T(\widetilde{x}))=\pi(\widetilde{x})$.

Daí, para $\widetilde{x} \in \widetilde{M}, \pi(\widetilde{I}(t, \widetilde{x}))=\pi(\widetilde{I}(t, T(\widetilde{x})))$. Logo existe $T_{t} \in G$ tal que $\widetilde{I}(t, T(\widetilde{x}))=T_{t}(\widetilde{I}(t, \widetilde{x}))$. Como $\widetilde{I}$ é contínua e $G$ é grupo discreto, segue que $T_{t}=T_{0}=T$, pois $T(\widetilde{x})=\widetilde{T}(0, T(\widetilde{x}))=T_{0}(\widetilde{I}(0, \widetilde{x}))=T_{0}(\widetilde{x})$.

Assim, $\widetilde{I}(t, T(\widetilde{x}))=T(\widetilde{I}(t, \widetilde{x}))$. Tomando $t=1$, segue que para todo $T \in G$, todo $\widetilde{x} \in \widetilde{M}, \widetilde{f}(T(\widetilde{x}))=\widetilde{I}(1, T(\widetilde{x}))=T(\widetilde{I}(1, \widetilde{x}))=T(\widetilde{f}(\widetilde{x}))$. 
A conta acima mostra que quando tomamos o levantamento $\widetilde{f}$ de $f$ dado por uma isotopia da identidade com a $f$, temos que $\tilde{f}$ comuta com as transformações de recobrimento, e assim, estamos dentro das hipóteses do teoria equivariante de Brouwer.

Um caminho $\gamma:[a, b] \rightarrow \operatorname{dom}(I)$ é positivamente transverso para uma folheação $\mathcal{F}$ se acontecer o seguinte: Para cada $t \in[a, b]$, existe uma vizinhança $W$ de $\gamma(t)$ e uma carta $h: W \rightarrow(0,1)^{2}$ compatível com a orientação enviando a folheação restrita $\left.\mathcal{F}\right|_{W}$ sobre a folheação vertical orientada para baixo tal que se $\pi_{1}$ é a projeção na primeira coordenada em $\mathbb{R}^{2}$, então a aplicação $\pi_{1}(h(\gamma))$ é crescente em uma vizinhança de $t$ (Veja figura 5). Em todo esse texto, a menos de menção em contrário, transverso significará positivamente transverso. Intuitivamente, dizer que $\gamma$ é transverso significa que localmente o caminho cruza da direita para a esquerda.

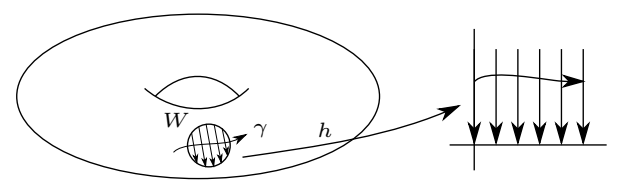

Figura 5: $\gamma$ é positivamente transverso para $\mathcal{F}$.

Se $f: \mathbb{R}^{2} \rightarrow \mathbb{R}^{2}$ é um homeomorfismo de Brouwer que comuta com $G=$ $\operatorname{Deck}(M)$, então pelo teorema equivariante, existe $\mathcal{F}$ uma folheação $G$ invariante formada por linhas de Brouwer. Le Calvez ([13]) também mostra que para cada $x \in \mathbb{R}^{2}$, existe um caminho $\gamma$ transverso para $\mathcal{F}$ que une $x$ à $f(x)$. A demonstração é simples e faremos em seguida. Considere $W$ o conjunto dos pontos $y \in \mathbb{R}^{2}$ tal que existe um caminho transverso para $\mathcal{F}$ que une $x$ a $y$. Claramente $W$ é um conjunto aberto não vazio e sua fronteira é formada pela folha $\phi_{x}$ e possivelmente por outras folhas $\phi$ tal que $\bar{W} \subseteq \bar{L}(\phi)$. Se o Ponto $f(x)$ não pertence a $W$, então deve existir alguma $\phi$ tal que $f(x) \in \bar{R}(\phi)$. Mas $\phi$ é linha de Brouwer e consequentemente, $f^{-1}(\bar{R}(\phi)) \subseteq \bar{R}(\phi)$. Mas $f(x) \in \bar{R}(\phi)$ e $x=f^{-1}(f(x)) \notin \bar{R}(\phi)$, o que é um absurdo. Logo $f(x) \in W$ e portanto, existe um caminho transverso de $x$ a $f(x)$. Reciprocamente, se uma folheação do plano é tal que para todo $x$ existe um caminho transverso de $x$ a $f(x)$, segue que as folhas são linhas de Brouwer para $f$. 
Considere agora $f: M \rightarrow M$ um homeomorfismo isotópico à identidade em uma superfície orientável $M$. Seja $I=\left(I_{t}\right)_{t \in[0,1]}$ uma isotopia maximal e defina $\widetilde{f}=\left(\widetilde{I}_{t}\right)_{t \in[0,1]}$ o levantamento de $f$ dado pela isotopia no espaço de recobrimento universal $\widetilde{\operatorname{dom}}(I)$ de $\operatorname{dom}(I)$. Se $V$ é uma componente conexa de $\widetilde{\operatorname{dom}}(I), V$ é um plano topológico e $\widetilde{f}$ comuta com as transformações de recobrimento e é livre de pontos fixos, visto que $I$ é maximal. Logo exite $\widetilde{\mathcal{F}}_{V}$ folheação de $V$ por linhas de Brouwer para $\tilde{f}$. Fazendo isso para cada componente conexa de $\widetilde{\operatorname{dom}}(I)$, utilizando a projeção $\pi: \widetilde{\operatorname{dom}}(I) \rightarrow \operatorname{dom}(I)$, podemos $\operatorname{definir} \operatorname{dom}(\mathcal{F})=$ $\pi\left(\cup_{V}\left(\widetilde{\mathcal{F}}_{V}\right)\right)$ e $\operatorname{sing}(\mathcal{F})=\operatorname{sing}(I)$. Assim, existe uma folheação $\mathcal{F}$ em $M$ cujo conjunto singular é exatamente igual ao conjunto singular da isotopia. Feito isso, temos a seguinte versão do teorema da folheação de Brouwer-Le Calvez $(\operatorname{veja}([13]))$ :

Teorema 2.8. Seja $f: M \rightarrow M$ é um homeomorfismo isotópico à identidade em uma superfície orientável $M$. Se I é uma isotopia maximal para $f$, então existe uma folheação singular orientada $\mathcal{F}$ sobre $M$ tal que o conjunto singular $\operatorname{sing}(\mathcal{F})$ coincide com $\operatorname{fix}(I)$ e para todo $x \in \operatorname{dom}(I)$, a trajetória $I(x)$ é homotópica em $\operatorname{dom}(I)$, relativo aos fins, a um caminho $\gamma$ transverso para $\mathcal{F}$.

Diremos que $\mathcal{F}$ é transversa para $I$ caso as hipóteses do teorema anterior sejam satisfeitas. A segunda parte do teorema vem do fato que para cada $\widetilde{x}$ no levantamento, existe um caminho transverso que une $\widetilde{x}$ a $\widetilde{f}(\widetilde{x})$. Este caminho é homotópico com extremos fixos, à trajetória $\widetilde{I}(\widetilde{x})$ dado pela isotopia. Assim, basta projetar em $M$ e temos que a trajetória $I(x)$ é homotópica, com extremos fixos, a um caminho transverso para $\mathcal{F}$.

Agora se $f, M$ e $I$ são como no teorema acima, então $\mathcal{F}$ pode ser levantada para uma folheação $\widetilde{\mathcal{F}}$ não singular de $\widetilde{\operatorname{dom}}(I)$, e tomando $\widetilde{f}$ o levantamento de $f$ dado pela isotopia $I$, pela segunda parte do teorema, segue que cada folha em $\widetilde{\operatorname{dom}}(I)$ é uma linha de Brouwer para $\widetilde{f}$.

Uma observação técnica antes de prosseguir para a próxima seção: em todo este trabalho, não faremos distinção entre $\gamma_{1}:[a, b] \rightarrow \operatorname{dom}(\mathrm{I})$ e $\gamma_{2}:[a, b] \rightarrow M$ tais que $\gamma_{1}(t)=\gamma_{2}(t)$, para todo $t \in[a, b]$. Embora rigorosamente sejam caminhos diferentes, iremos tratar ambos como sendo o mesmo caminho. 


\section{Forcing theory}

Nessa seção vamos exibir a teoria de forcing para trajetórias transversas em homeomorfismos de superfície desenvolvida por Le Calvez e Tal, que vem permitindo a decoberta de novos resultados (veja por exemplo ([16]) e ([19])).

Sejam $f, M, I$ e $\mathcal{F}$ como no Teorema 2.8. Dadas $\gamma_{i}:[a, b] \rightarrow \operatorname{dom}(I)$, i $=1$, 2, duas curvas transversas em $M$, diremos que $\gamma_{1}$ é $\mathcal{F}$ equivalente a $\gamma_{2}$, se existem levantamentos $\widetilde{\gamma}_{1}$ e $\widetilde{\gamma}_{2}$ de $\gamma_{1}$ e $\gamma_{2}$ em $\widetilde{\operatorname{dom}}(I)$ respectivamente tal que $\gamma_{1}$ e $\gamma_{2}$ cruzam as mesmas folhas. Denotaremos $\gamma_{1} \sim_{\mathcal{F}} \gamma_{2}$ e caso não haja confusão, também denotaremos $\gamma_{1} \sim \gamma_{2}$. Essa relação acima é uma relação de equivalência, e, para facilitar a notação, denotaremos a classe de equivalência de uma curva $\gamma$ como sendo $\gamma$, ou seja, não faremos distinção entre a classe e os seus representantes. A definição acima não é equivalente a definir $\gamma_{1}$ e $\gamma_{2}$ cruzando as mesmas folhas em dom(I). A figura (6) é um exemplo dado por Tal e Le Calvez ([16]) em que $p_{1}, p_{2}$ e $p_{3} \in \operatorname{Fix}(I), \gamma_{1}$ e $\gamma_{2}$ são caminhos transversos à folheação que cruzam as mesmas folhas em $\operatorname{dom}(I)$ que não são equivalentes.

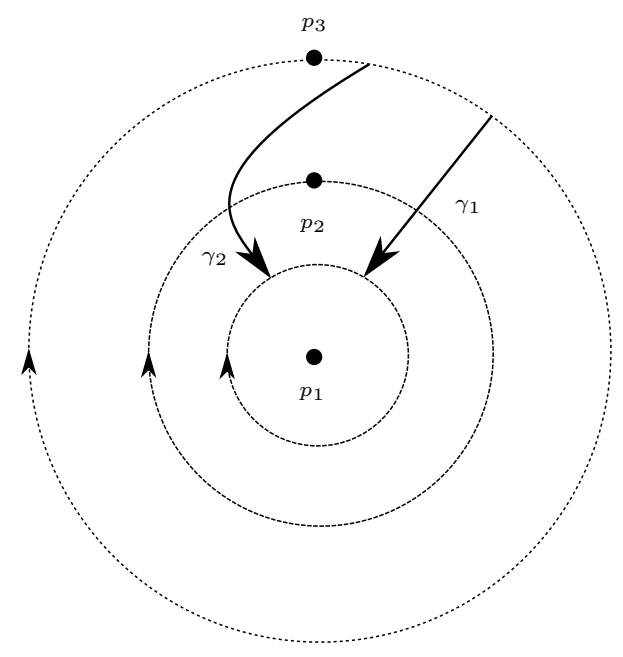

Figura 6: $\gamma_{1}$ e $\gamma_{2}$ cruzam as mesmas folhas mas não são equivalentes.

Pelo teorema 2.8, para cada $x$ em $\operatorname{dom}(I)$, a trajetória $I(x)$ é homotópica com extremos fixos a um caminho transverso $\gamma$. Se $\widetilde{\gamma}$ é um levantamento de $\gamma$ em $\widetilde{\operatorname{dom}}(I)$, então qualquer outro caminho $\mathcal{F}$ equivalente a $\gamma$, com extremos em $\widetilde{x}$ e $\widetilde{f}(\widetilde{x})$ satisfaz o mesmo. Isso nos sujere a seguinte definição: Denotaremos por 
$I_{\mathcal{F}}(x)$ a classe de caminhos transversos que ligam $x$ a $f(x)$ e que são homotópicos a $I(x)$ em $\operatorname{dom}(I)$ com extremos fixos. O conjunto $I_{\mathcal{F}}(x)$ é chamado de a trajetória transversa de $x$ e é não vazio pelo teorema 2.8. Como nessa teoria não importará qual represenante da classe escolhermos, podemos supor que $I_{\mathcal{F}}(x)$ denota qualquer elemento de sua classe.

Para cada $n \geq 1$, definimos a trajetória transversa n-ésima de $x$, que denoratemos por $I_{\mathcal{F}}^{n}(x)$, da seguinte forma: Um caminho transverso $\gamma$ pertence a essa classe se e somente se for homotópico a $I^{n}(x)$ em $\operatorname{dom}(I) \operatorname{com} x, f(x), \ldots, f^{n}(x)$ fixos. Podemos definir de forma análoga $I_{\mathcal{F}}^{\mathbb{Z}}(x)$, a trajetória transversa completa de $x$. Novamente, como não faz diferença em qual elemento da classe estaremos tomando, podemos supor que $I_{\mathcal{F}}^{n}(x)$ e $I_{\mathcal{F}}^{\mathbb{Z}}(x)$ representam qualquer elemento de suas classes de equivalência respectivamente, lembrando que pelo teorema 2.8 estas classes não são vazias.

Definição 3.1. Um caminho transverso $\gamma:[a, b] \rightarrow \operatorname{dom}(I)$ é n-admissivel se é $\mathcal{F}$ equivalente ao caminho $I_{\mathcal{F}}^{n}(x)$, para algum $x \in \operatorname{dom}(I)$.

Denotaremos por $\phi_{x}$ a folha que contém $x$. Se $\gamma:[a, b] \rightarrow \operatorname{dom}(I)$ é um caminho transverso n-admissível, e se tomarmos $\widetilde{\gamma}:[a, b] \rightarrow \widetilde{\operatorname{dom}}(I)$ um levantamento de $\gamma$, então existe $\widetilde{x}$ um levantamento de $x$ tal que $\widetilde{x} \in \phi_{\widetilde{\gamma}(a)}$ e $\widetilde{f}^{n}(\widetilde{x}) \in \phi_{\widetilde{\gamma}(b)}$.

Estamos quase aptos a enunciar o teorema de forcing para trajetórias transversas, mas primeiro, precisamos entender o conceito de intersecção transversa. Para isto, necessitamos da seguinte definição (Veja figura 7):

Definição 3.2. Seja $\gamma_{i}: \mathbb{R} \rightarrow \mathbb{R}^{2}$ uma linha, com $i \in\{0,1,2\}$. Dizemos que $\gamma_{2}$ está acima de $\gamma_{1}$ com relação à $\gamma_{0}$ (ou que $\gamma_{1}$ está abaixo de $\gamma_{2}$ com relação à $\left.\gamma_{0}\right)$ se forem satisfeitos:

(i) as três linhas são duas a duas disjuntas;

(ii) nenhuma das linhas separa as outras duas;

(iii) se $\lambda_{1}, \lambda_{2}$ são dois caminhos disjuntos ligando $z_{1}=\gamma_{0}\left(t_{1}\right), z_{2}=\gamma_{0}\left(t_{2}\right)$ a $z_{1}^{\prime} \in \gamma_{1}, z_{2}^{\prime} \in \gamma_{2}$, respectivamente, e não intersectam as linhas, exceto pelos extremos, então $t_{2}>t_{1}$. 


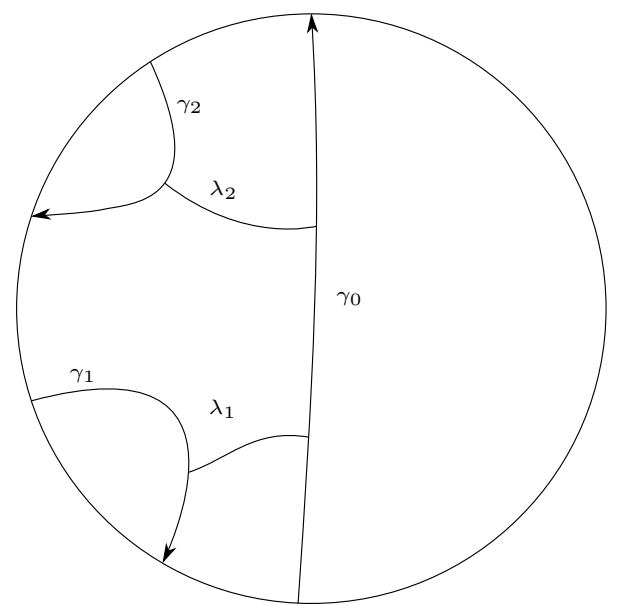

Figura 7: $\gamma_{2}$ está acima de $\gamma_{1}$ relativo a $\gamma_{0}$.

Observe que a definição de acima (ou abaixo) não depende das direções de $\gamma_{1}$ e $\gamma_{2}$, apenas da de $\gamma_{0}$. Vejamos agora a definição de intersecção transversa. Considere primeiramente o caso em que $M=\mathbb{R}^{2}$ e $\mathcal{F}$ sem singularidades. Relembre que $\phi_{x}$ denota a folha de $\mathcal{F}$ que contém $x$.

Definição 3.3. Sejam $\gamma_{1}: J_{1} \rightarrow \mathbb{R}^{2}$ e $\gamma_{2}: J_{2} \rightarrow \mathbb{R}^{2}$ dois caminhos transversos definidos em intervalos $J_{1}$ e $J_{2}$ respectivamente, tais que $\gamma_{1}\left(t_{1}\right)=\gamma_{2}\left(t_{2}\right)$. Diremos que $\gamma_{1}$ intersecta $\mathcal{F}$ transversalmente $\gamma_{2}$ em $\gamma_{1}\left(t_{1}\right)=\gamma_{2}\left(t_{2}\right)$ se existirem $a_{1}, b_{1} \in$ $J_{1}$ com $a_{1}<t_{1}<b_{1}$ e $a_{2}, b_{2} \in J_{2}$ com $a_{2}<t_{2}<b_{2}$ tais que vale

(i) $\phi_{\gamma_{2}\left(a_{2}\right)}$ está abaixo de $\phi_{\gamma_{1}\left(a_{1}\right)}$ com relação à $\phi_{\gamma_{1}\left(t_{1}\right)}$;

(ii) $\phi_{\gamma_{2}\left(b_{2}\right)}$ está acima de $\phi_{\gamma_{1}\left(b_{1}\right)}$ com relação à $\phi_{\gamma_{1}\left(t_{1}\right)}$,

ou

(i') $\phi_{\gamma_{2}\left(a_{2}\right)}$ está acima de $\phi_{\gamma_{1}\left(a_{1}\right)}$ com relação à $\phi_{\gamma_{1}\left(t_{1}\right)}$;

(ii') $\phi_{\gamma_{2}\left(b_{2}\right)}$ está abaixo de $\phi_{\gamma_{1}\left(b_{1}\right)}$ com relação à $\phi_{\gamma_{1}\left(t_{1}\right)}$.

No caso geral, diremos que dois caminhos transversos $\gamma_{1}$ e $\gamma_{2}$ se intersectam $\mathcal{F}$ transversalmente em $\gamma_{1}\left(t_{1}\right)=\gamma_{2}\left(t_{2}\right)$ se existem levantamentos destes caminhos para o recobrimento universal $\widetilde{\operatorname{dom}}(I)$, e tais levantamentos satisfazem a definição anterior (Veja figura 8). Se por acaso $\gamma=\gamma_{1}=\gamma_{2}$, diremos que $\gamma$ tem 


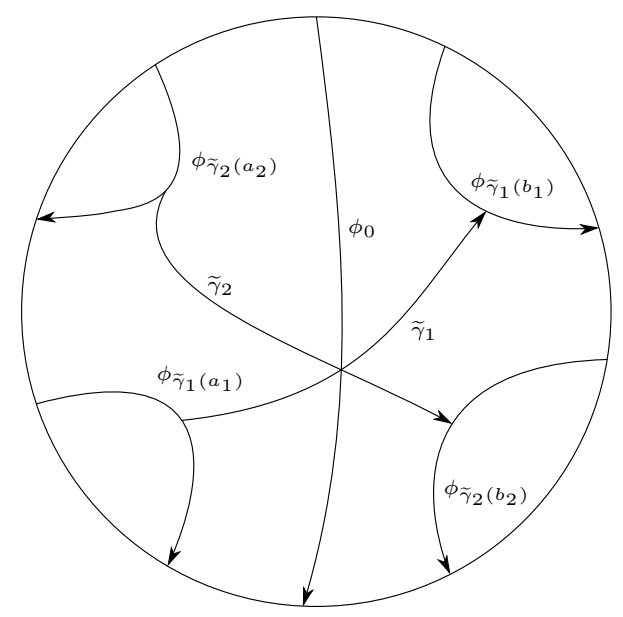

Figura 8: $\gamma_{1}$ tem uma $\mathcal{F}$ intersecção transversa com $\gamma_{2}$.

uma $\mathcal{F}$ autointersecção transversa se $\gamma$ possui dois levantamentos que possuem uma intersecção transversa. Caso $\gamma_{1}$ tenha uma $\mathcal{F}$ intersecção transversa com $\gamma_{2}$, e $\gamma_{1} \sim_{\mathcal{F}} \gamma_{3}$ para algum caminho $\gamma_{3}$, segue que $\gamma_{2}$ tem uma $\mathcal{F}$ intersecção transversa com $\gamma_{3}$, ou seja, a propriedade de intersecção transversa não depende do representante da classe de equivalência que se está utilizando.

Sejam dois caminhos transversos $\gamma_{1}: J_{1} \rightarrow M$ e $\gamma_{2}: J_{2} \rightarrow M$ para intervalos $J_{1}$ e $J_{2}$, e sejam $t_{1} \in J_{1}$ e $t_{2} \in J_{2}$ tais que $\gamma_{1}\left(t_{1}\right)=\gamma_{2}\left(t_{2}\right)$. Denifimos o caminho transverso $\gamma_{3}=\left.\left.\gamma_{1}\right|_{\left[a_{1}, t_{1}\right]} \gamma_{2}\right|_{\left[t_{2}, b_{2}\right]}$ concatenando os caminhos $\left.\gamma_{1}\right|_{\left[a_{1}, t_{1}\right]}$ e $\left.\gamma_{2}\right|_{\left[t_{2}, b_{2}\right]}$. Ou seja, existe um intervalo $J_{3}$ e um ponto $t_{3} \in J_{3}$ tal que para todo $t \in J_{3}$, $\gamma_{3}(t)=\gamma_{1}(t)$ se $t \leq t_{3}$ e $\gamma_{3}(t)=\gamma_{2}(t)$ se $t \geq t_{3}$. Vejamos agora o teorema de forcing:

Teorema $3.4([16])$. Suponha que $\gamma_{i}:\left[a_{i}, b_{i}\right] \rightarrow M, i \in\{1,2\}$ são dois caminhos transversos que se intersectam $\mathcal{F}$ transversalmente em $\gamma_{1}\left(t_{1}\right)=\gamma_{2}\left(t_{2}\right)$. Se $\gamma_{1}$ é admissível de ordem $n_{1}$ e $\gamma_{2}$ é admissível de ordem $n_{2}$, então $\left.\left.\gamma_{1}\right|_{\left[a_{1}, t_{1}\right]} \gamma_{2}\right|_{\left[t_{2}, b_{2}\right]}$ e $\left.\left.\gamma_{2}\right|_{\left[a_{2}, t_{2}\right]} \gamma_{1}\right|_{\left[t_{1}, b_{1}\right]}$ são admissíveis de ordem $n_{1}+n_{2}$. Além disso, ou um dos caminhos é admissivel de ordem $\min \left(n_{1}, n_{2}\right)$ ou ambos são admissíveis de ordem $\max \left(n_{1}, n_{2}\right)$.

Corolário 3.5. Nas notações do teorema anterior, se $\gamma_{1}=\gamma_{2}$ for admissível de ordem n, então $\left.\left.\gamma_{1}\right|_{\left[a_{1}, t_{1}\right]} \gamma_{2}\right|_{\left[t_{2}, b_{2}\right]}$ ou $\left.\left.\gamma_{2}\right|_{\left[a_{2}, t_{2}\right]} \gamma_{1}\right|_{\left[t_{1}, b_{1}\right]}$ é admissivel de ordem $n$.

O teorema acima é relativamente fácil de se demonstrar, entretanto, está se 
demonstrando uma incrível ferramenta para a demonstração de novos teoremas com inúmeras aplicações. Como exemplo, com este trabalho e o trabalho precedente de Le Calvez e Tal ([19]), é possível caracterizar e dar uma estimativa mínima para entropia de uma aplicação isotópica à identidade em termos das trajetetóras transversas. A teoria de forcing para trajetórias transversas está se demonstrando um ótimo instrumento para a obtenção de resultados relativos à teoria dos conjuntos de rotação (veja [14] para mais detalhes sobre o conjunto de rotação do toro e [16] para alguns destes novos resultados). No próximo capítulo faremos o uso da teoria de forcing para melhorar a estimativa de Le Calvez e Tal em ([19]) para a entropia topológica para uma certa classe de homeomorfismos de superfícies isotópicos à identidade.

\section{Estimativa para a entropia}

\subsection{Resultado principal}

O principal resutado deste trabalho é o seguinte:

Teorema 4.1. Seja $M$ uma superfície orientada, $f$ um homeomorfismo de $M$ isotópico à identidade, I uma isotopia maximal de $f$ e $\mathcal{F}$ uma folheação transversa para $I$. Se $\gamma:[a, b] \rightarrow \operatorname{dom}(I)$ é um caminho admissivel de ordem $n e$ se $\gamma$ tem uma autointersecção transversa, então a entropia de $f$ é pelo menos $\log (2) / n$.

O teorema acima melhora a estimativa de mínima entropia topológica de $f$ do trabalho dos pesquisadores Le Calvez e Tal ([19] Teorema N), que provaram que a entropia topológica de $f$, com as mesmas hipóteses do Teorema 4.1, deve ser de no mínimo $\log (4) /(3 n)$. O fato interessante sobre essa nova estimativa é que os pesquisadores deste trabalho acrediram que a estimativa $\log (2) / n$ é ótima, entretanto, não foi encontrado um exemplo que comprove esta afirmação.

Dizemos que $f$ tem $k>0$ intersecções transversas em relação a uma deck $T$ em $\widetilde{\operatorname{dom}}(I)$ se existe um levantamento $\widetilde{\gamma}$ de $\gamma$ em $\widetilde{\operatorname{dom}}(I)$ que possua intersecção transversa $\operatorname{com} T^{i}(\widetilde{\gamma})$ para $i=1, \ldots, k$. Veja figura 9 . 


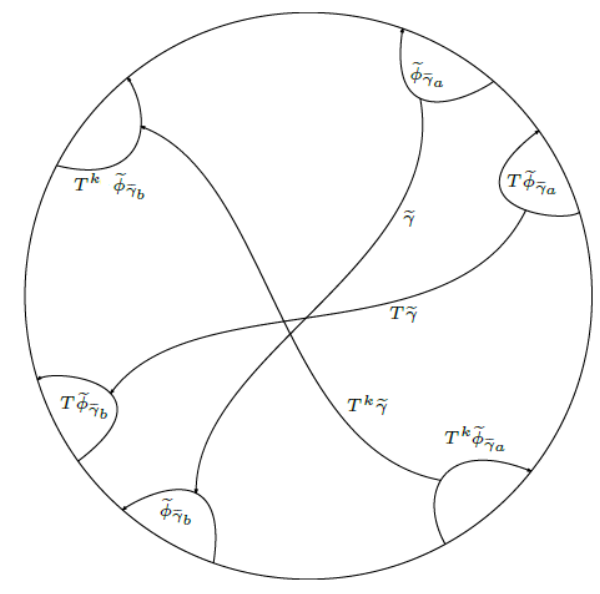

Figura 9: $\widetilde{\gamma} \operatorname{com} k=3$ intersecções transversas em relação a $T$.

Uma pergunta natural que surge do teorema 4.1 é se existe um resultado equivalente para quando $f$ tenha $k>0$ intersecções transversas em relação a uma deck T. Assim, temos a seguinte conjectura:

Conjectura 4.2. Seja $M$ uma superfície orientada, $f$ um homeomorfismo de $M$ isotópico à identidade, I uma isotopia maximal de $f$ e $\mathcal{F}$ uma folheação transversa para I. Se $\gamma:[a, b] \rightarrow \operatorname{dom}(I)$ é um caminho admissivel de ordem $n$ e se $\gamma$ tem $k>0$ autointersecções transversas em relação a uma deck $T$ de $\widetilde{\operatorname{dom}}(I)$, então a entropia de $f$ é pelo menos $\log (2 k) / n$.

Observe que o Teorema 4.1 é um caso particular da conjectura 4.2 quando $k=1$. Para demostrar o Teorema 4.1 é suficiente mostrar para o caso em que $\gamma$ é admissível de ordem 1, pois se $\gamma$ for $n$ admissível para $f$, então segue que $\gamma$ é 1 admissível para $f^{n}$, daí o resultado segue pois $h(f)=h\left(f^{n}\right) / n$.

Exemplo: Considere a figura 10. Na imagem da esquerda temos o plano com uma folheação para uma aplicação $f$ com exatamente dois ponto fixos para a isotopia $I$ e também temos um caminho transverso $\gamma$ que podemos supor admissível de ordem 1. Na imagem da direita temos o espaço de recobrimento universal $\widetilde{\operatorname{dom}}(I)$ com a folheação levantada. Nele existe um levantamento $\widetilde{\gamma}$ de $\gamma$ e uma transformação de recobrimento $T$ tal que $\widetilde{\gamma}$ e $T(\widetilde{\gamma})$ possuem uma intersecção transversa, o que mostra que $\gamma$ tem uma autointersecção transversa. 

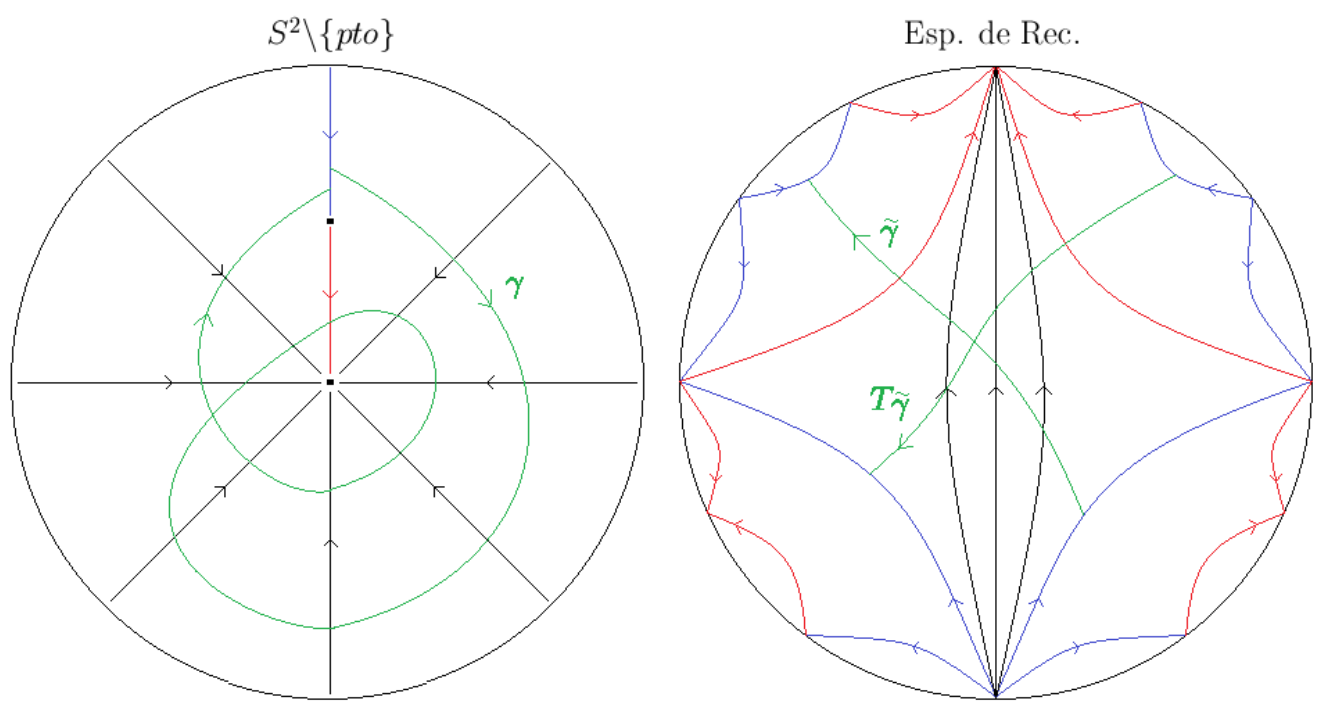

Figura 10: $\gamma$ tem uma autointersecção transversa.

Segue então que estamos nas hipóteses do teorema 4.1 e portanto, a entropia topológica de $f$ é ao menos $\log (2)$.

Embora seja apenas uma conjectura, muitos dos resultados feitos para $k=1$ também valem quando $k>1$. Por isto vamos supor, por enquanto, que $k>0$ é qualquer.

Vamos fixar as seguintes notações:

- $M$ é uma superfície orientada (sem bordo, não necessariamente compacta e não necessariamente de tipo finito);

- $f$ é um homeomorfismo de $M$ isotópico à identidade;

- $I=(I)_{t \in[0,1]}$ é uma isotopia maximal entre $f$ e a identidade;

- $\mathcal{F}$ é uma folheação transversa para $I$; 
- O conjunto $\operatorname{dom}(I)$ é o domínio de $I$ e seu espaço de recobrimento universal é denotado por $\widetilde{\operatorname{dom}}(I)$.

- $\gamma$ é um caminho transverso admissível de ordem 1 com uma autointersecção transversa;

- $\widetilde{I}$ é o levantamento da isotopia $I$ em $\widetilde{\operatorname{dom}}(I)$;

- $\widetilde{f}$ é o levantamento de $f$ em $\widetilde{\operatorname{dom}}(I)$ dado pela isotopia $\widetilde{I}$;

- $\widetilde{\mathcal{F}}$ é o levantamento de $\mathcal{F}$ em $\widetilde{\operatorname{dom}}(I)$;

- $\widetilde{\gamma}$ é um levantamento de $\gamma$ em $\widetilde{\operatorname{dom}}(I)$;

- T é uma transformação de recobrimento tal que $\widetilde{\gamma}$ e $T(\widetilde{\gamma})$ possuam uma $\widetilde{\mathcal{F}}$ intersecção transversa em $T\left(\widetilde{\gamma}\left(s_{1}\right)\right)=\widetilde{\gamma}\left(t_{1}\right)$, com $s_{1}<t_{1}$.

A ideia da prova será construir um conjunto ilimitado $\widetilde{Q}$ em $\widetilde{\operatorname{dom}}(I)$ que se comportará praticamente como uma ferradura topológica a menos de uma translação por uma deck. Usaremos essa deck para obtermos um espaço de recobrimento anular e a partir dele, uma esfera topológica. Desta forma, considerando o homeomorfismo induzido na esfera topológica, conseguimos uma semiconjugação com o shift em $k$ símbolos. Para obtermos essa estimativa para $f$, devido o conjunto $\widetilde{Q}$ ser ilimitado, temos que fazer uma aproximação, e no limite desta, teremos a estimativa desejada.

Vejamos algumas notações que serão úteis no decorrer do texto. Se $\widetilde{x} \in$ $\widetilde{\operatorname{dom}}(I)$, denotaremos por $\phi_{\widetilde{x}}$ a folha de $\widetilde{\mathcal{F}}$ que contém $\widetilde{x}$ e por $\phi_{x}$ a folha de $\mathcal{F}$ que contém $x \in \operatorname{dom}(I)$. Denotaremos por $\phi_{\tilde{x}}^{+}$a semifolha positiva que contém $\widetilde{x}$ e por $\phi_{\tilde{x}}^{-}$a semifolha negativa que contém $\widetilde{x}$. Observe que se $T^{\prime}$ é uma transformação de recobrimento então $T^{\prime}\left(\phi_{\widetilde{x}}\right)=\phi_{T^{\prime} \widetilde{x}}$.

É possível colocar uma ordem parcial nos pontos de uma folha da seguinte forma: Se $\widetilde{x}_{1}, \widetilde{x}_{2} \in \widetilde{\operatorname{dom}}(I)$ com $\widetilde{x}_{2} \in \phi_{\widetilde{x}_{1}}^{+}$, então diremos que $\widetilde{x}_{1} \leq \widetilde{x}_{2}$. Caso $\widetilde{x}_{1} \neq \widetilde{x}_{2}$, diremos que $\widetilde{x}_{1}<\widetilde{x}_{2}$. Neste caso, $\phi_{\widetilde{x}_{1}, \widetilde{x}_{2}}$ denota o segmento conexo contido em $\phi_{\widetilde{x}_{1}}$ cujos extremos são $\widetilde{x}_{1}$ e $\widetilde{x}_{2}$. Observe que $T^{\prime}\left(\phi_{\widetilde{x}_{1}, \widetilde{x}_{2}}\right)=\phi_{T^{\prime} \widetilde{x}_{1}, T^{\prime} \widetilde{x}_{2}}$.

Seja $\phi$ uma folha e $S$ uma deck qualquer em $\widetilde{\operatorname{dom}}(I)$. Se $R(\phi)$ e $R(S(\phi))$ 
são disjuntos, então $R\left(S^{j}(\phi)\right), j \in \mathbb{Z}$ são dois a dois disjuntos. O mesmo vale para $L\left(S^{j}(\phi)\right), \overline{R\left(S^{j}(\phi)\right)}$ e $\overline{L\left(S^{j}(\phi)\right)}, j \in \mathbb{Z}$. Com efeito, pelo fato que $S$ é um homeomorfismo de $\widetilde{\operatorname{dom}}(I)$ que preserva orientação sem pontos fixos, e portanto um homeomorfismo de Brouwer, temos que $S(R(\widetilde{\phi}))=R(S(\widetilde{\phi}))$. Daí, utilizando o lema dos discos livres (lema 2.4), obtemos o resultado. A demonstração é análoga para os outros casos.

Fabio Tal e Patrice Le Calvez fazem em ([19]) uma construção do espaço de recobrimento anular e demonstraram que exite $k_{1}>0$ tal que $T^{-k_{1}}\left(\phi_{\widetilde{\gamma}(b)}\right)$ está acima de $\phi_{\widetilde{\gamma}(a)}$ e abaixo de $T\left(\phi_{\widetilde{\gamma}(a)}\right)$ relativo a uma linha $\gamma^{*}$. Resumiremos essa construção a seguir no próximo lema:

Lema 4.3. Existe uma linha $\gamma^{*}$ satisfazendo $\gamma^{*}(t+1)=T\left(\gamma^{*}(t)\right)$ e existe $k_{1}>0$ inteiro, tal que $T^{-k_{1}}\left(\phi_{\widetilde{\gamma}(b)}\right)$ está acima de $\phi_{\widetilde{\gamma}(a)}$ e abaixo de $T\left(\phi_{\widetilde{\gamma}(a)}\right)$ relativo a $\gamma^{*}$. Além disso, se $i<j$, então $T^{i}\left(\phi_{\widetilde{\gamma}(a)}\right)$ está abaixo de $T^{j}\left(\phi_{\widetilde{\gamma}(a)}\right)$ relativo a $\gamma^{*}$. O mesmo vale para $\phi_{\widetilde{\gamma}(b)}$.

Demonstração. Desde que $\widetilde{\gamma}$ e $T(\widetilde{\gamma})$ tem uma intersecção transversa, os conjuntos $R\left(\phi_{\widetilde{\gamma}(a)}\right), R\left(T\left(\phi_{\widetilde{\gamma}(a)}\right)\right), L\left(\phi_{\widetilde{\gamma}(b)}\right)$ e $L\left(T\left(\phi_{\widetilde{\gamma}(b)}\right)\right)$ são todos disjuntos.

Considere o espaço de recobrimento anular $\widehat{\operatorname{dom}}(I)=\widetilde{\operatorname{dom}}(I) / T$. Denote por $\pi_{1}: \widetilde{\operatorname{dom}}(I) \rightarrow \widehat{\operatorname{dom}}(I)$ a projeção de recobrimento, por $\hat{I}$ a isotopia induzida, por $\hat{f}$ o levantamento de $f$ induzido, por $\widehat{\mathcal{F}}$ a folheação induzida e por $\widehat{\gamma}$ a projeção de $\widetilde{\gamma}$.

O fato que $R\left(\phi_{\widetilde{\gamma}(a)}\right)$ e $R\left(T\left(\phi_{\widetilde{\gamma}(a)}\right)\right)$ são disjuntos implica que $\phi_{\widehat{\gamma}(a)}$, a projeção de $\phi_{\widetilde{\gamma}(a)}$, é homoclínica para um fim de $\widehat{\operatorname{dom}}(I)$, ou seja, começa e termina no mesmo fim, e que os conjuntos $R\left(T^{k}\left(\phi_{\widetilde{\gamma}(a)}\right)\right), k \in \mathbb{Z}$, são dois a dois disjuntos.

Da mesma forma, como $L\left(\phi_{\widetilde{\gamma}(b)}\right)$ e $L\left(T\left(\phi_{\widetilde{\gamma}(b)}\right)\right)$ são disjuntos implica que $\phi_{\widehat{\gamma}(b)}$, a projeção de $\phi_{\widetilde{\gamma}(b)}$, é homoclínica para um fim de $\widehat{\operatorname{dom}}(I)$ e que os conjuntos $L\left(T^{j}\left(\phi_{\widetilde{\gamma}(b)}\right)\right), j \in \mathbb{Z}$, são dois a dois disjuntos.

Desde que $T(\widetilde{\gamma})$ e $\widetilde{\gamma}$ tem uma $\widetilde{\mathcal{F}}$ intersecção transversa segue que os dois finais citados são iguais. Caso contrário, poderíamos encontrar um caminho $\delta$ conectando $\phi_{\widetilde{\gamma}(a)}$ e $\phi_{\widetilde{\gamma}(b)}$, contido em $L\left(T^{j}\left(\phi_{\widetilde{\gamma}_{a}}\right)\right)$ e $R\left(T^{j^{\prime}}\left(\phi_{\widetilde{\gamma}_{b}}\right)\right)$, para todo $j, j^{\prime} \in \mathbb{Z}$, se projetando sobre um caminho simples de $\widehat{\operatorname{dom}}(I)$ e, tal que $\delta \cap T(\delta)=\emptyset$. Para encontrar $\delta$ basta tomar um caminho simples contido em $\overline{L\left(\phi_{\widehat{\gamma}_{a}}\right)}$ e $\overline{R\left(\phi_{\widehat{\gamma}_{b}}\right)}$ que saí de $\phi_{\widehat{\gamma}(a)}$ e chega em $\phi_{\widehat{\gamma}(b)}$ se rotacionando, no recobrimento anular, a quantidade 
de vezes necessária para que no levantamento do anel, ele chegue em $\phi_{\widetilde{\gamma}(b)}$. Veja figura 11. Este final comum será denotado por $N$ e o outro por $S$. Observe que $R\left(T^{j}\left(\phi_{\widetilde{\gamma}(a)}\right)\right) \cap L\left(T^{j^{\prime}}\left(\phi_{\widetilde{\gamma}(b)}\right)\right)=\emptyset, \forall j, j^{\prime} \in \mathbb{Z}$.
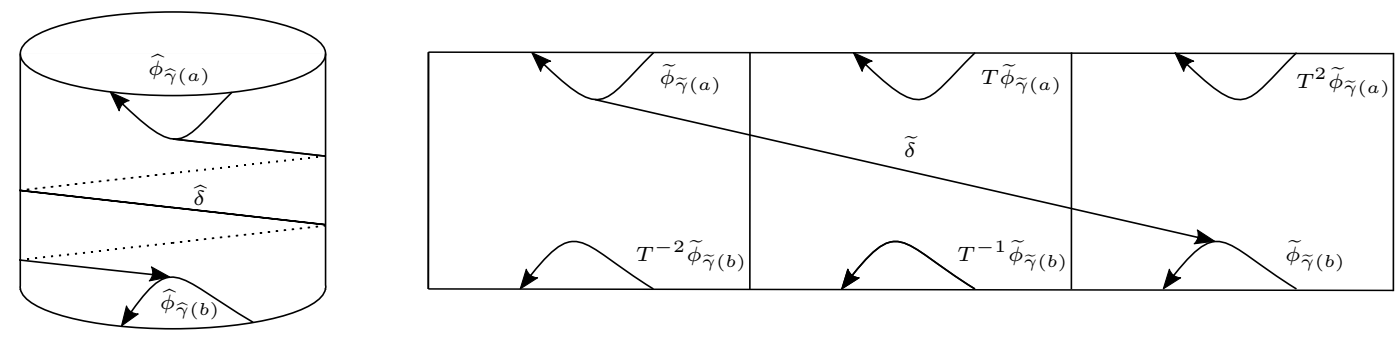

Figura 11: Exemplo da existência de $\delta$.

O complemento de $R\left(\phi_{\widehat{\gamma}(a)}\right) \cup L\left(\phi_{\widehat{\gamma}(b)}\right)$ é um conjunto aberto essencial de $\widehat{\operatorname{dom}}(I)$, ou seja, existe alguma curva não contrátil neste conjunto. Considere então $\Gamma^{*}$ um loop essencial simples nesse complemento que é levantado para uma linha $\gamma^{*}: \mathbb{R} \rightarrow \widetilde{\operatorname{dom}}(I)$, satisfazendo $\gamma^{*}(t+1)=T\left(\gamma^{*}(t)\right), \forall t \in \mathbb{R}$. Daí existe um único $k_{1} \geq 1$ tal que $T^{-k_{1}}\left(\phi_{\widetilde{\gamma}(b)}\right)$ está acima de $\phi_{\widetilde{\gamma}(a)}$ mas abaixo de $T\left(\phi_{\widetilde{\gamma}(a)}\right)$ relativo a $\gamma^{*}$.
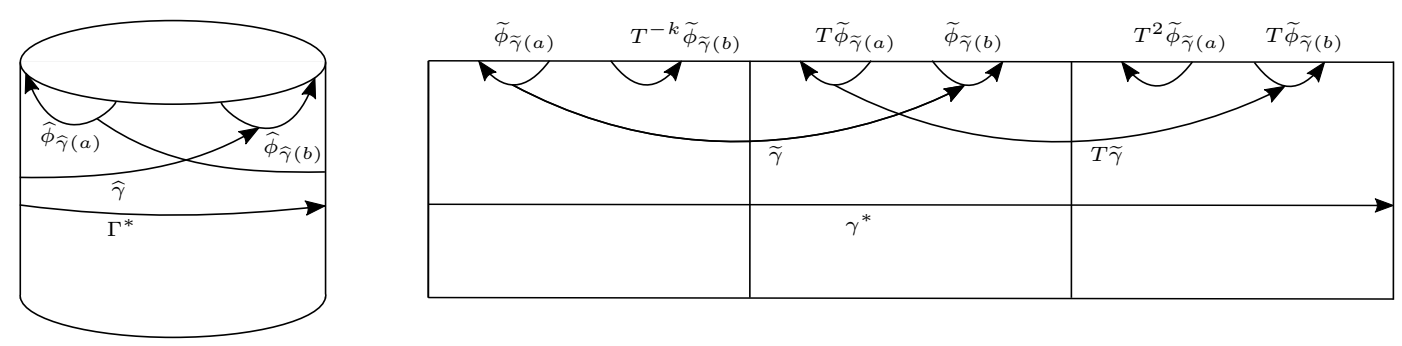

Figura 12: Exemplo com $k=1$.

O inteiro $k_{1}$ pode ser definido pelo fato que $\widetilde{\gamma}$ e $T^{j}(\widetilde{\gamma})$ tem $\widetilde{\mathcal{F}}$ intersecção transversa se $1 \leq j \leq k_{1}$ e não tem intersecção transversa se $j>k_{1}$. Observe também que pela construção de $\gamma^{*}$, se $i<j$, então $T^{i}\left(\phi_{\widetilde{\gamma}(a)}\right)$ está abaixo de $T^{j}\left(\phi_{\widetilde{\gamma}(a)}\right)$ relativo a $\gamma^{*}$ e isso conclui o lema.

Por conveniência, vamos denotar $\widetilde{\phi}_{a}=\phi_{\widetilde{\gamma}(a)}$ e $\widetilde{\phi}_{b}=T^{-k_{1}}\left(\phi_{\widetilde{\gamma}(b)}\right)$. Vamos supor que $\widetilde{\gamma}$ e $T(\widetilde{\gamma})$ tenham uma intersecção transversa em $T\left(\widetilde{\gamma}\left(s_{1}\right)\right)=\widetilde{\gamma}\left(t_{1}\right)$ com 
$s_{1}<t_{1}$. Podemos fazer isso pois caso $s_{1}>t_{1}$ poderiamos tomar a deck $T^{-1} \mathrm{em}$ vez da deck $T$. É importante ressaltar que não podemos ter $s_{1}=t_{1}$, pois assim $T$ teria um ponto fixo.

Lema 4.4. Existe uma curva $\gamma_{k}$ equivalente a $\gamma$ e um levantamento $\widetilde{\gamma}_{k}$ de $\gamma_{k}$ tal que $\widetilde{\gamma}_{k}$ tem uma $\mathcal{F}$ intersecção transversa com $T^{i}\left(\widetilde{\gamma}_{k}\right)$ em $T^{i}\left(\widetilde{\gamma}_{k}\left(s_{i}\right)\right)=\widetilde{\gamma}_{k}\left(t_{i}\right)$, para $i=1, \ldots, k$, com $s_{k}<\ldots<s_{1}<t_{1}<\ldots<t_{k}$ e $\widetilde{\gamma}_{k}$ intersecta $T^{i}\left(\widetilde{\gamma}_{k}\right)$ apenas nos pontos citados acima, para $i=1, \ldots, k$.

Demonstração. Observe que existe um caminho $\widetilde{\gamma}_{1}:[a, b] \rightarrow \widetilde{\operatorname{dom}}(I)$ equivalente a $\widetilde{\gamma}$ tal que $\widetilde{\gamma}_{1}$ e $T \widetilde{\gamma}_{1}$ tem uma $\mathcal{F}$ intersecção transversa em $T\left(\widetilde{\gamma}\left(s_{1}\right)\right)=\widetilde{\gamma}\left(t_{1}\right)$ com $s_{1}<t_{1}$ e este é o único ponto de intersecção de $\widetilde{\gamma}_{1}$ e $T(\widetilde{\gamma})$.

Caso $k \geq 2, \widetilde{\gamma}_{1}$ tem uma intersecção transversa com $T\left(\widetilde{\gamma}_{1}\right)$ e $T^{2}\left(\widetilde{\gamma}_{1}\right)$, e além disso, $T\left(\widetilde{\gamma}_{1}\right)$ tem uma intersecção transversa com $T^{2}\left(\widetilde{\gamma}_{1}\right)$. Pela construção que fizemos antes, $\widetilde{\gamma}_{1}$ e $T\left(\widetilde{\gamma}_{1}\right)$ tem apenas um único ponto de intersecção, assim, aplicando a deck $T$, o mesmo vale para $T\left(\widetilde{\gamma}_{1}\right)$ e $T^{2}\left(\widetilde{\gamma}_{1}\right)$.

Afirmação: $\widetilde{\gamma}_{1}\left(\left[a, t_{1}\right]\right)$ não intersecta $T^{2}\left(\widetilde{\gamma}_{1}\right)$. Com efeito, como $\widetilde{\gamma}_{1}$ e $T\left(\widetilde{\gamma}_{1}\right)$ tem apenas um único ponto de intersecção, segue que $T\left(\widetilde{\gamma}_{1}\right)$ e $T^{2}\left(\widetilde{\gamma}_{1}\right)$ tem apenas um único ponto de intersecção em $T^{2}\left(\widetilde{\gamma}\left(s_{1}\right)\right)=T\left(\widetilde{\gamma}\left(t_{1}\right)\right)$, $s_{1}<t_{1}$. Como $\widetilde{\gamma}_{1}\left(t_{1}\right)=$ $T\left(\widetilde{\gamma}\left(s_{1}\right)\right) \in T\left(\widetilde{\gamma}_{1}\right)$ e $s_{1}<t_{1}$, segue que $\widetilde{\gamma}_{1}\left(t_{1}\right) \notin T^{2}\left(\widetilde{\gamma}_{1}\right)$. Daí, caso exista algum ponto nessa intersecção, ele deve estar no intervalo aberto $\left(a, t_{1}\right)$. Vamos mostrar que isto também não é possível. Suponha então que exista um ponto $a<w_{1}<t_{1}$ tal que $\widetilde{\gamma}_{1}\left(w_{1}\right) \in T^{2}\left(\widetilde{\gamma}_{1}\right)$. Observe que $\overline{R\left(\phi_{T\left(\widetilde{\gamma}_{1}(a)\right)}\right)} \cup T\left(\widetilde{\gamma}_{1}\right) \cup \overline{L\left(\phi_{T\left(\widetilde{\gamma}_{1}(b)\right)}\right)}$ divide $\widetilde{\operatorname{dom}}(I)$ em duas componentes conexas, com $T^{2}\left(\widetilde{\gamma}_{1}\left(\left[a, s_{1}\right)\right)\right)$ em uma e $\widetilde{\gamma}_{1}\left(\left[a, t_{1}\right)\right)$ em outra. Portanto, deve existir um $w_{2} \geq s_{1}$ tal que $\widetilde{\gamma}_{1}\left(w_{1}\right)=T^{2}\left(\widetilde{\gamma}_{1}\left(w_{2}\right)\right)$. Considere o caminho $\Gamma$ dado por $\left.\left.\left.\left(T^{2}\left(\widetilde{\gamma}_{1}\right)\right)\right|_{\left[s_{1}, w_{2}\right]}\left(\widetilde{\gamma}_{1}\right)\right|_{\left[w_{1}, t_{1}\right]}\left(T\left(\widetilde{\gamma}_{1}\right)\right)\right|_{\left[s_{1}, t_{1}\right]}$. Observe que $\Gamma$ é um caminho transverso e fechado, o que é um absurdo, pois assim a folheação $\mathcal{F}$ teria ao menos um ponto singular, o que não ocorre, e isto encerra a demonstração da afirmação (Veja figura 13).

Agora, veja que $\widetilde{\gamma}_{1}$ e $T^{2}\left(\widetilde{\gamma}_{1}\right)$ tem uma intersecção transversa, mas não possuem intersecção em $\widetilde{\gamma}_{1}\left(\left[a, t_{1}\right]\right)$. Assim, deve existir $a<s_{2}<s_{1}$ e $t_{1}<t_{2}<b$ tal que $\widetilde{\gamma}_{1}$ e $T^{2}\left(\widetilde{\gamma}_{1}\right)$ tem uma intersecção transversa em $T^{2}\left(\widetilde{\gamma}_{1}\left(s_{2}\right)\right)=\widetilde{\gamma}_{1}\left(t_{2}\right)$. Além disso, existe um trecho $\widetilde{\gamma}^{\prime}$ equivalente a $\widetilde{\gamma}_{1}\left(\left[t_{2}, b\right]\right)$ tal que este intersecta $T^{2}\left(\widetilde{\gamma}_{1}\right)$ apenas em $\widetilde{\gamma}_{1}\left(t_{2}\right)$ e não intersecta $T\left(\widetilde{\gamma}_{1}\right)$. Assim, se denotarmos $\widetilde{\gamma}_{2}$ o caminho 


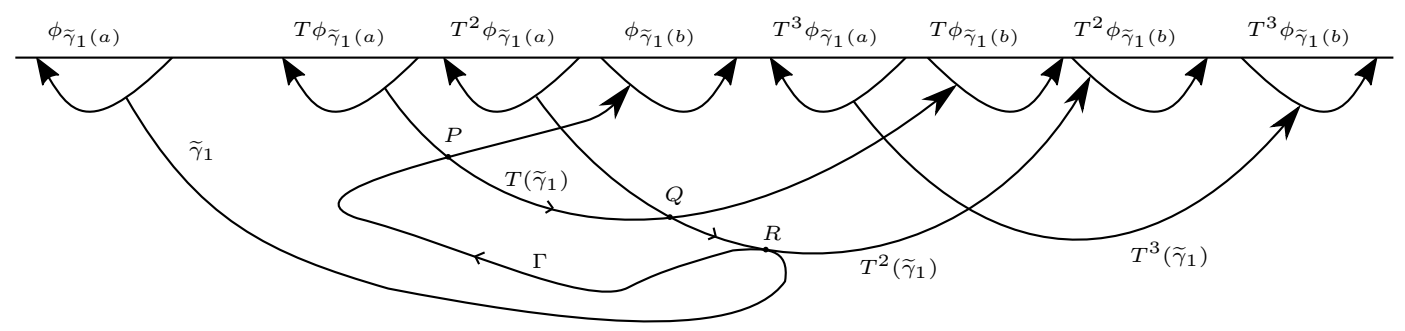

Figura 13: $P=\widetilde{\gamma}_{1}\left(t_{1}\right)=T\left(\widetilde{\gamma}_{1}\left(s_{1}\right)\right), Q=T\left(\widetilde{\gamma}_{1}\left(t_{1}\right)\right)=T^{2}\left(\widetilde{\gamma}_{1}\left(s_{1}\right)\right)$ e $R=\widetilde{\gamma}_{1}\left(w_{1}\right)=$ $T^{2}\left(\widetilde{\gamma}_{1}\left(w_{2}\right)\right)$. O caminho transverso $\Gamma$ formado pelos caminhos que unem $P, Q$ e $R$ é um caminho fechado, o que implicaria em pontos singulares na folheação, o que não pode ocorrer

equivalente a $\widetilde{\gamma}_{1}$ substituindo o trecho $\widetilde{\gamma}_{1}\left(\left[t_{2}, b\right]\right)$ por $\widetilde{\gamma}^{\prime}$ citado acima, resulta que $\widetilde{\gamma}_{2}$ tem uma intersecção transversa com $T\left(\widetilde{\gamma}_{2}\right)$ em $T\left(\widetilde{\gamma}_{2}\left(t_{1}\right)\right)=\widetilde{\gamma}_{2}\left(s_{1}\right)$ e com $T^{2}\left(\widetilde{\gamma}_{2}\right)$ em $T^{2}\left(\widetilde{\gamma}_{2}\left(t_{2}\right)\right)=\widetilde{\gamma}_{2}\left(s_{2}\right)$ (veja figura 14$)$.

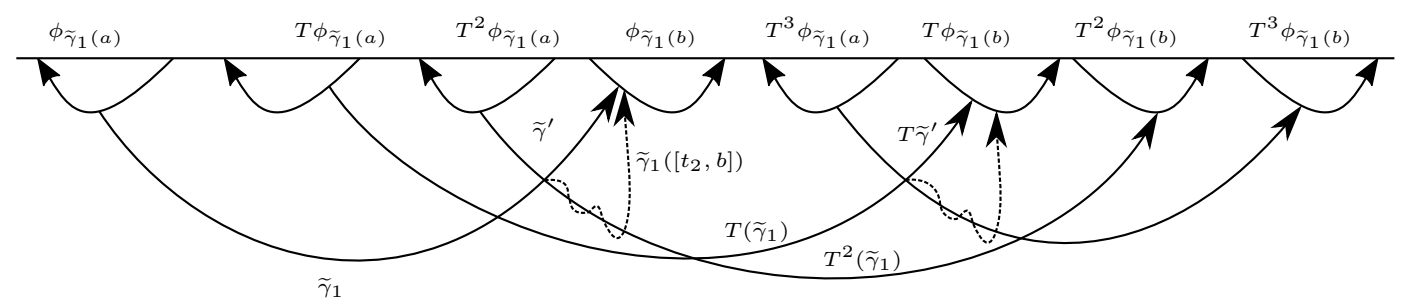

Figura 14: $\widetilde{\gamma}^{\prime}$ é equivalente a $\widetilde{\gamma}_{1}\left(\left[t_{2}, b\right]\right)$ e este intersecta $T^{2}\left(\widetilde{\gamma}_{1}\right)$ apenas em $\widetilde{\gamma}_{1}\left(t_{2}\right)$ e não intersecta $T\left(\widetilde{\gamma}_{1}\right)$.

Portanto, $\widetilde{\gamma}_{2}$ é $\mathcal{F}$ equivalente a $\widetilde{\gamma}$ e tem uma $\mathcal{F}$ intersecção transversa com $T^{i}\left(\widetilde{\gamma}_{2}\right)$ em $T^{i}\left(\widetilde{\gamma}_{2}\left(s_{i}\right)\right)=\widetilde{\gamma}_{2}\left(t_{i}\right)$, para $i=1,2$, com $s_{2}<s_{1}<t_{1}<t_{2}$ e intersecta $T\left(\widetilde{\gamma}_{2}\right)$ e $T^{2}\left(\widetilde{\gamma}_{2}\right)$ apenas nos pontos citados acima.

Suponha agora que exista $\widetilde{\gamma}_{p}$ equivalente a $\widetilde{\gamma}$, com $k \geq p>0$, e que $\widetilde{\gamma}_{p}$ tem uma intersecção transversa com $T^{i} \widetilde{\gamma}$ em $T^{i}\left(\widetilde{\gamma}\left(s_{i}\right)\right)=\widetilde{\gamma}\left(t_{i}\right)$, para $i=1, \ldots, p$, com $s_{p}<\ldots<s_{1}<t_{1}<\ldots<t_{p}$. Se $k \geq p+1$, então podemos usar um argumento semelhante ao que fizemos acima, trocando o segmento $\gamma_{p}\left(\left[t_{p+1}, b\right]\right)$ por outro equivalente, e construir $\widetilde{\gamma}_{p+1}$ com propriedades análogas a $\widetilde{\gamma}_{p}$.

Para simplificar a notação, vamos continuar a chamar a curva equivalente 
$\gamma_{k}$ do lema anterior de $\gamma$, pois a propriedade de intersecção transversa é uma propriedade comum a todas as curvas em uma classe $\mathcal{F}$ equivalentes e o que será importante neste texto é que como $\gamma$ é 1-admissível, então $\gamma_{k}$ é também 1-admissível.

Observe que pela construção acima, e pelo fato que as intersecções transversas são invariantes por automorfismos de recobrimento, ou seja, a imagem por uma deck $S$ de duas curvas que possuem uma intersecção transversa gera duas curvas que possuem intersecção transversa, então se $0<j-i \leq k, i$ e $j$ inteiros, segue que $T^{i}(\widetilde{\gamma})$ tem uma intersecção transversa com $T^{j}(\widetilde{\gamma})$ em $T^{j}\left(\widetilde{\gamma}\left(s_{j-i}\right)\right)=$ $T^{i}\left(\widetilde{\gamma}\left(t_{j-i}\right)\right)$.

Para cada $0<i \leq k$, sabemos que $\widetilde{\gamma}$ tem uma intersecção transversa com $T^{i}(\widetilde{\gamma})$ em $T^{i}\left(\widetilde{\gamma}\left(s_{i}\right)\right)=\widetilde{\gamma}\left(t_{i}\right)$. Além disso, ambos são admissíveis de ordem 1 . Considere:

$$
\widetilde{\gamma}_{3, i}=\left.\left.\widetilde{\gamma}\right|_{\left[a, t_{i}\right]} T^{i} \widetilde{\gamma}\right|_{\left[s_{i}, b\right]}
$$

e

$$
\widetilde{\gamma}_{4, i}=\left.\left.T^{i} \widetilde{\gamma}\right|_{\left[a, s_{i}\right]} \widetilde{\gamma}\right|_{\left[t_{i}, b\right]}
$$

Pelo teorema de Forcing, um dos caminhos definidos acima deve ser admissível de ordem 1. De fato, temos o seguinte lema:

Lema 4.5. O caminho $\widetilde{\gamma}_{4, i}$ é admissível de ordem 1 .

A demonstração deste lema pode ser encontrada em [16], proposição 23 (Veja figura 15).

Observe que $\widetilde{f}\left(T^{-i}\left(\widetilde{\phi}_{a}\right)\right)$ intersecta $\widetilde{\phi}_{b}$ para cada $i=1, \ldots, k$.

Para construir o conjunto $\widetilde{Q}$ é importante notar o seguinte fato: sabemos, por hipótese, que $\widetilde{\gamma}$ e $T(\widetilde{\gamma})$ tem uma intersecção transversa em $T\left(\widetilde{\gamma}\left(s_{1}\right)\right)=\widetilde{\gamma}\left(t_{1}\right)$ com $s_{1}<t_{1}$. Por causa disto, ou $\widetilde{\phi}_{a}$ está acima de $T\left(\widetilde{\phi}_{a}\right)$ relativo a $\phi_{\widetilde{\gamma}\left(t_{1}\right)}$ ou $\widetilde{\phi}_{a}$ está abaixo de $T\left(\widetilde{\phi}_{a}\right)$ relativo a $\phi_{\widetilde{\gamma}\left(t_{1}\right)}$. Neste trabalho vamos supor que acontece este segundo caso, como é mostrado na figura 17. A construção matemática do conjunto de $\widetilde{Q}$ a partir do primeiro caso (veja figura 16) é análoga ao segundo caso. 


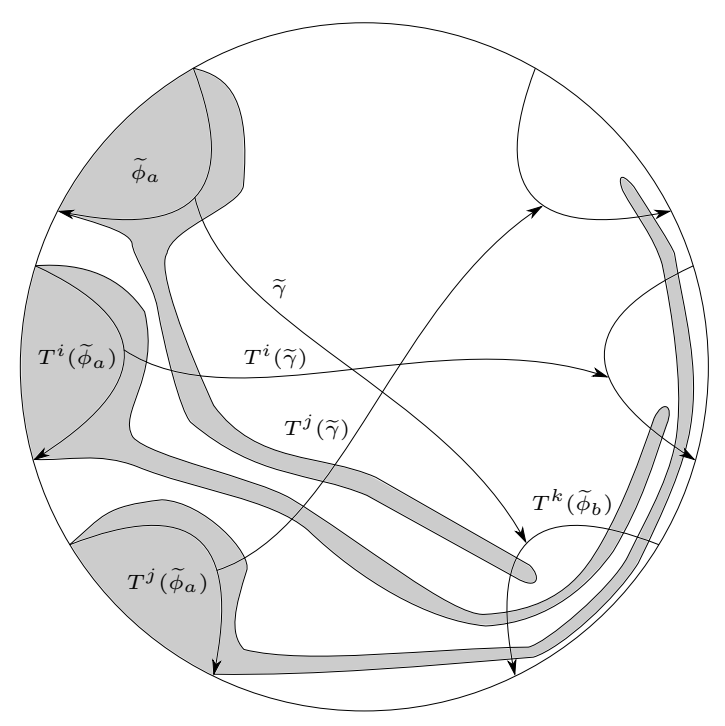

Figura 15: $\gamma_{4, i}$ é admissível de ordem 1.

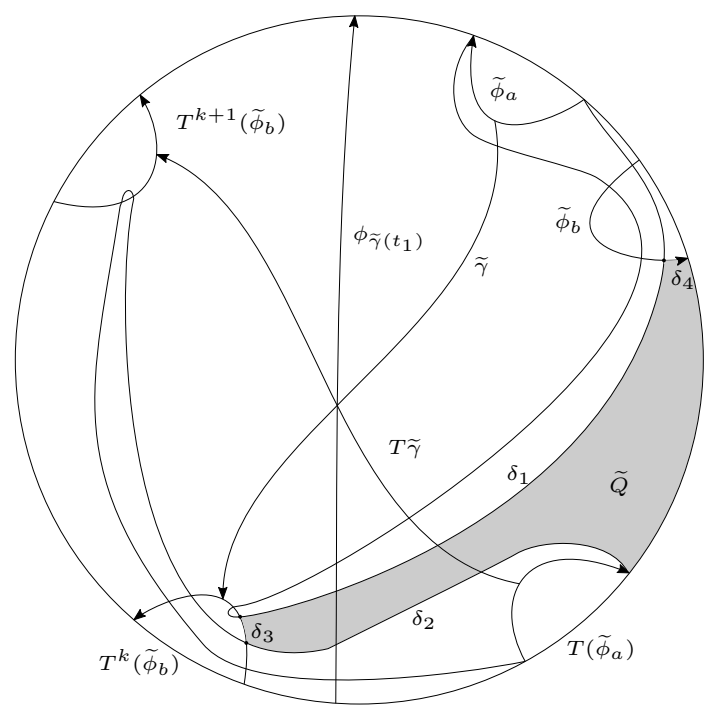

Figura 16: Conjunto $\widetilde{Q}$ quando $\widetilde{\phi}_{a}$ está abaixo de $T\left(\widetilde{\phi}_{a}\right)$ relativo a $\phi_{\widetilde{\gamma}\left(t_{1}\right)}$.

Vamos agora definir o conjunto $\widetilde{Q}$, que será um "retângulo" com um vértice no infinito e será fundamental para a estimativa da entropia de $f$.Como $\widetilde{\gamma}$ é 1 admissível, segue que $\widetilde{f}\left(\widetilde{\phi}_{a}\right)$ intersecta $T^{k}\left(\widetilde{\phi}_{b}\right)$. Além disso, deve intersectar também $\widetilde{\phi}_{b}$. Assim, existe pelo menos um segmento que conecta $\widetilde{\phi}_{b}$ e $T^{k}\left(\widetilde{\phi}_{b}\right)$ e está contido em $\widetilde{f}\left(\widetilde{\phi}_{a}\right)$. Seja $\delta_{1}$ um destes segmentos. Podemos supor que $\delta_{1}$ é o último segmento na ordem induzida pela orientação da folha $\widetilde{\phi}_{a}$, que $\delta_{1} \cap \widetilde{\phi}_{b}=\left\{p_{1}\right\}$ 
e $\delta_{1} \cap T^{k}\left(\widetilde{\phi}_{b}\right)=\left\{p_{2}\right\}$.

Existe um ponto $q_{3} \in T\left(\widetilde{\phi}_{a}\right)$ com $\tilde{f}\left(q_{3}\right) \in T^{k}\left(\widetilde{\phi}_{b}\right)$ tal que $\tilde{f}\left(\widetilde{\phi}_{q_{3}}^{-}\right) \cap T^{k}\left(\widetilde{\phi}_{b}\right)=$ $\left\{\widetilde{f}\left(q_{3}\right)\right\}$. Considete $p_{3}=\widetilde{f}\left(q_{3}\right)$ e seja $\delta_{2}=\widetilde{f}\left(\widetilde{\phi}_{q_{3}}^{-}\right)$. Seja $\delta_{3}$ o segmento em $T^{k}\left(\widetilde{\phi}_{b}\right)$ que conecta $p_{2}$ e $p_{3}$ e seja $\delta_{4}=\widetilde{\phi}_{p_{1}}^{-}$.

O complemento do conjunto $\delta_{1} \cup \delta_{2} \cup \delta_{3} \cup \delta_{4}$ divide $\widetilde{\operatorname{dom}}(I)$ em duas componentes conexas ilimitadas. Considere $\widetilde{Q}$ o fecho da componente conexa que não contém $\widetilde{\phi}_{a}$. Veja figura 17 .

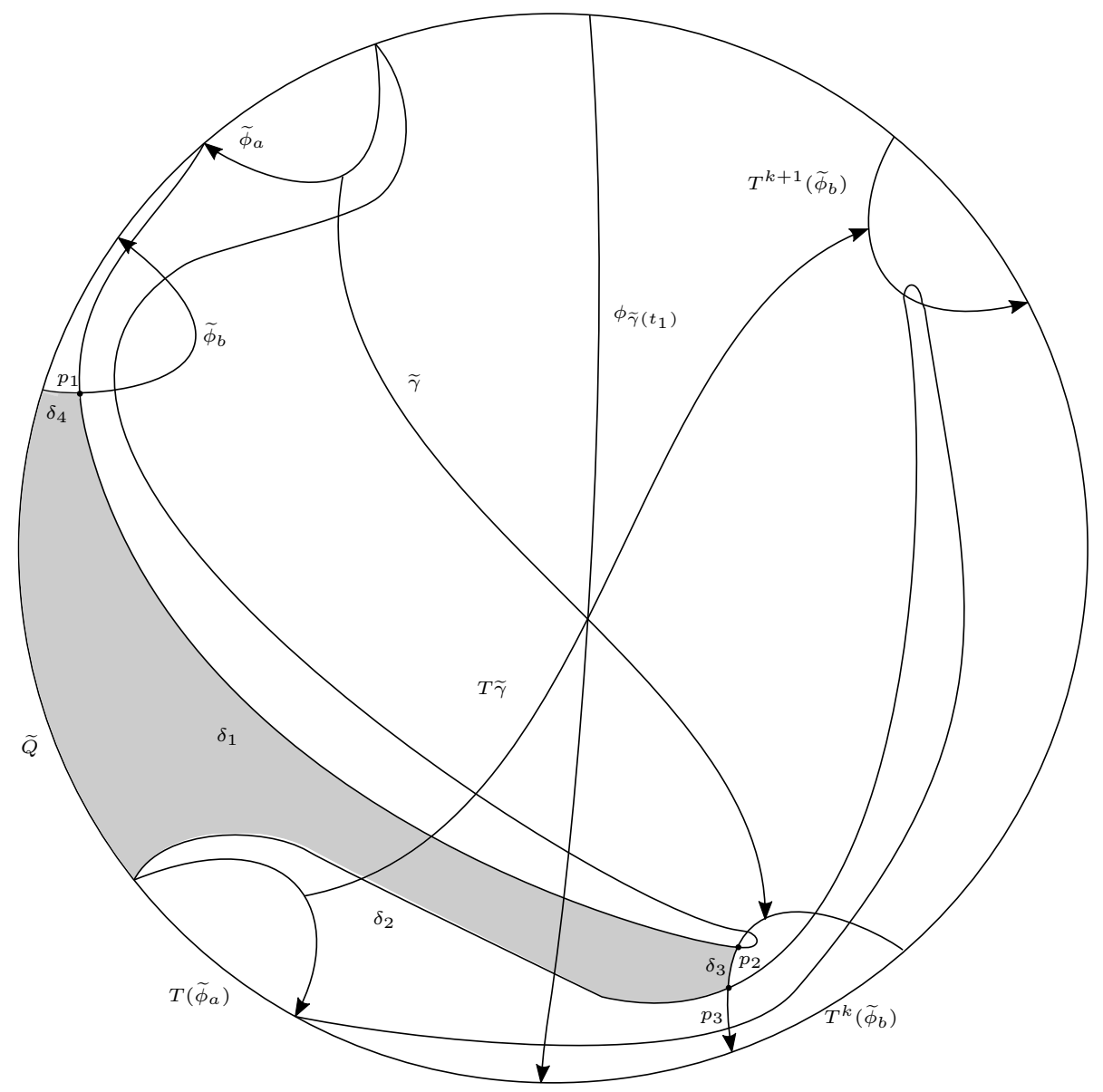

Figura 17: Conjunto $\widetilde{Q}$ quando $\widetilde{\phi}_{a}$ está acima de $T\left(\widetilde{\phi}_{a}\right)$ relativo a $\phi_{\widetilde{\gamma}\left(t_{1}\right)}$.

Observação 4.6. Para $\widetilde{Q}$ existir de fato como foi definido, $\widetilde{f}\left(T \widetilde{\phi}_{a}\right)$ não deve intersectar $\widetilde{\phi}_{b}$. Entretanto, eles realmente possuem intersecção vazia, pois caso contrário conseguiríamos encontrar uma curva fechada simples $\gamma^{\prime}$, formada por segmentos $A, B, C$ e $D$ com $A \subseteq \delta_{4}, B=\delta_{1}, C=\delta_{3}$ e $D \subseteq \delta_{2}$ tal que o índice 
desta curva por $\widetilde{f}^{-1}$ é não nulo, o que significaria que $\widetilde{f}^{-1}$, e portanto $\widetilde{f}$, teria um ponto fixo no disco topológico limitado por $\gamma^{\prime}$, o que é um absurdo (Veja figura 18). A demonstração deste fato é semelhante a parte da prova da proposição 12 de [19].

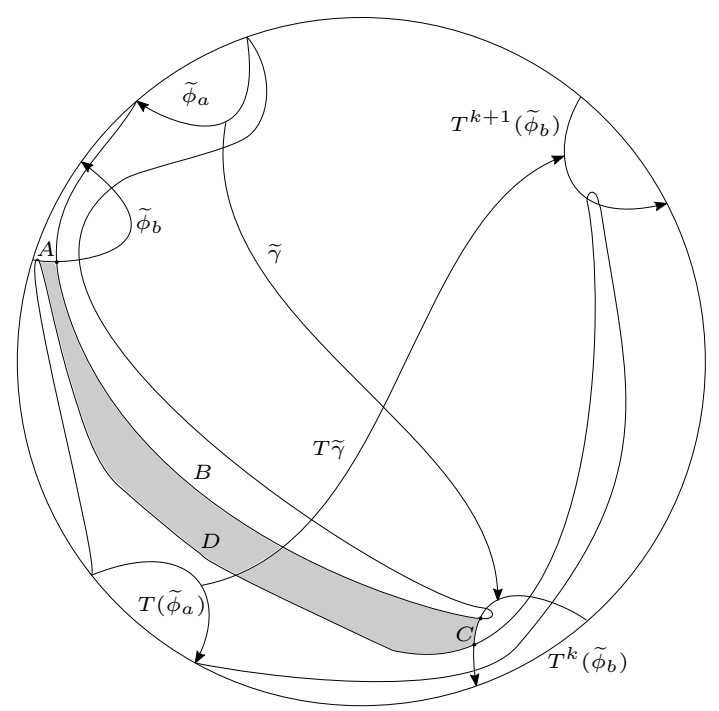

Figura 18: Segmentos $A, B, C$ e $D$

Proposição 4.7. Se $k=1$ então $\widetilde{Q} \cap T \widetilde{Q}=\emptyset$.

Demonstração. Para provar a proprosição, vamos mostrar que os conjuntos $\widetilde{Q}$ e $T(\widetilde{Q})$ não se intersectam na fronteira (Veja a figura 19). Trivialmente, $\delta_{i} \cap T\left(\delta_{i}\right)=$ $\emptyset$ para todo $i=1,2,3,4$. Vamos então demonstrar os outros casos:

- $\delta_{1} \cap T\left(\delta_{2}\right)=\emptyset$. Por definição, $T\left(\delta_{2}\right)$ está contido $\widetilde{f}\left(T^{2}\left(\widetilde{\phi}_{a}\right)\right)$ e $\delta_{1}$ está contido em $\widetilde{f}\left(\widetilde{\phi}_{a}\right)$, o que implica que a intersecção é disjunta.

- $\delta_{1} \cap T\left(\delta_{3}\right)=\emptyset$. Observe que $\delta_{1}$ é um caminho cujos extremos estão em $\widetilde{\phi}_{b}$ e em $T\left(\widetilde{\phi}_{b}\right)$ e intersecta estes conjuntos apenas nestes pontos. Afirmação: $\delta_{1}$ não intersecta $T^{j}\left(\widetilde{\phi}_{b}\right)$, para todo $j \geq 2$. Com efeito, suponha que sim, e que $p$ seja o maior valor para o qual vale essa propriedade. Então existe um subsegmento $\alpha$ de $\delta_{1}$ que intersecta $\widetilde{\phi}_{b}$ e $T^{p}\left(\widetilde{\phi}_{b}\right)$. Mas daí $T^{-1}(\alpha)$ não intersecta $\widetilde{f}\left(\widetilde{\phi}_{a}\right)$, o que implica que $T^{-1}(\alpha)$ deve intersectar $T^{p}\left(\widetilde{\phi}_{b}\right)$. Mas 
assim, $\alpha$ intesecta $T^{p+1}\left(\widetilde{\phi}_{b}\right)$, o que é um absurdo. Como $T\left(\delta_{3}\right)$ está contido em $T^{2}\left(\widetilde{\phi}_{b}\right)$, o resultado segue.

- $\delta_{1} \cap T\left(\delta_{4}\right)=\emptyset$. Veja que $\delta_{1}$ intersecta $T\left(\widetilde{\phi}_{b}\right)$ em apenas um ponto, e como é disjunto de $T\left(\delta_{1}\right)$, segue o resultado, pois $T\left(\delta_{4}\right)$ e $\delta_{1}$ estão em diferentes componente conexa do complementar de $L\left(T\left(\widetilde{\phi}_{b}\right)\right) \cup \delta_{1} \cup L\left(T^{(2)}\left(\widetilde{\phi}_{b}\right)\right)$.

- $\delta_{2} \cap T\left(\delta_{1}\right)=\emptyset$. Por definição, $\delta_{2}=\widetilde{f}\left(\widetilde{\phi}_{q_{3}}^{-}\right) \subseteq \widetilde{f}\left(T\left(\widetilde{\phi}_{a}\right)\right)$, onde $q_{3}$ é o primeiro ponto em $T\left(\widetilde{\phi}_{a}\right)$, na ordem induzida em $T\left(\widetilde{\phi}_{a}\right)$, tal que $p_{3}=\widetilde{f}\left(q_{3}\right) \in T\left(\widetilde{\phi}_{b}\right)$. Observe que $T\left(\delta_{1}\right)$ também está contido em $\widetilde{f}\left(T\left(\widetilde{\phi}_{a}\right)\right)$, e que $T\left(\delta_{1}\right) \cap T\left(\widetilde{\phi}_{b}\right)=$ $\left\{T\left(p_{1}\right)\right\}$, que é um ponto diferente diferente de $p_{3}$, pois caso contrário, teríamos uma intersecção de $\delta_{1} \operatorname{com} T\left(\delta_{1}\right)$, o que não pode ocorrer.

- $\delta_{2} \cap T\left(\delta_{3}\right)=\emptyset$. Pelo mesmo argumento da afirmação feita no item $\delta_{1} \cap T\left(\delta_{2}\right)$, é possível mostrar que $\delta_{2}$ não intersecta $T^{2}\left(\widetilde{\phi}_{b}\right)$, logo também não intersecta $T\left(\delta_{3}\right) \subseteq T^{2}\left(\widetilde{\phi}_{b}\right)$.

- $\delta_{2} \cap T\left(\delta_{4}\right)=\emptyset$. Observe que $\delta_{2} \cap T\left(\widetilde{\phi}_{b}\right)=\left\{p_{3}\right\}$ e $T\left(\delta_{4}\right) \subseteq T\left(\widetilde{\phi}_{b}\right)$. Mas $\left\{p_{3}\right\}$ não intersecta $T\left(\delta_{4}\right)$, pois caso contrário, teríamos que ter uma interesecção de $\delta_{2}$ com $\delta_{1}$, o que não ocorre.

- $\delta_{3} \cap T\left(\delta_{1}\right)=\emptyset$. Veja que $T\left(\delta_{1}\right)$ intersecta $T\left(\widetilde{\phi}_{b}\right)$ em um único ponto. Caso esta interesecção não fosse vazia, $T\left(\delta_{1}\right)$ necessariamente deveria intersectar $\delta_{1}$ ou intersectar pelo menos um segundo ponto em $T\left(\widetilde{\phi}_{b}\right)$, o que é um absurdo.

- $\delta_{3} \cap T\left(\delta_{2}\right)=\emptyset$. Pelo mesmo argumento da observação 4.6, segue que $T\left(\delta_{2}\right)$ não intersecta $T\left(\widetilde{\phi}_{b}\right)$.

- $\delta_{3} \cap T\left(\delta_{4}\right)=\emptyset$. Caso não fosse vazia a intersecção, então necessariamente $\delta_{1}$ deveria intersectar $T\left(\delta_{1}\right)$, o que é um absurdo.

- $\delta_{4} \cap T\left(\delta_{1}\right)=\emptyset$. Segue do fato que $T\left(\delta_{1}\right)$ não intersecta $\widetilde{f}\left(\widetilde{\phi}_{a}\right)$ e intersecta $T\left(\widetilde{\phi}_{b}\right)$ apenas em um único ponto.

- $\delta_{4} \cap T\left(\delta_{2}\right)=\emptyset$. Pelo fato de que $T\left(\delta_{2}\right)$ não intersecta $T\left(\delta_{4}\right) \cup T\left(\delta_{1}\right)$, o resultado segue. 
- $\delta_{4} \cap T\left(\delta_{3}\right)=\emptyset$. Veja que $\delta_{4}$ está contido em $\widetilde{\phi}_{b}$ e $T\left(\delta_{3}\right)$ está contido em $T^{2}\left(\widetilde{\phi}_{b}\right)$, e assim, o resultado é trivial.

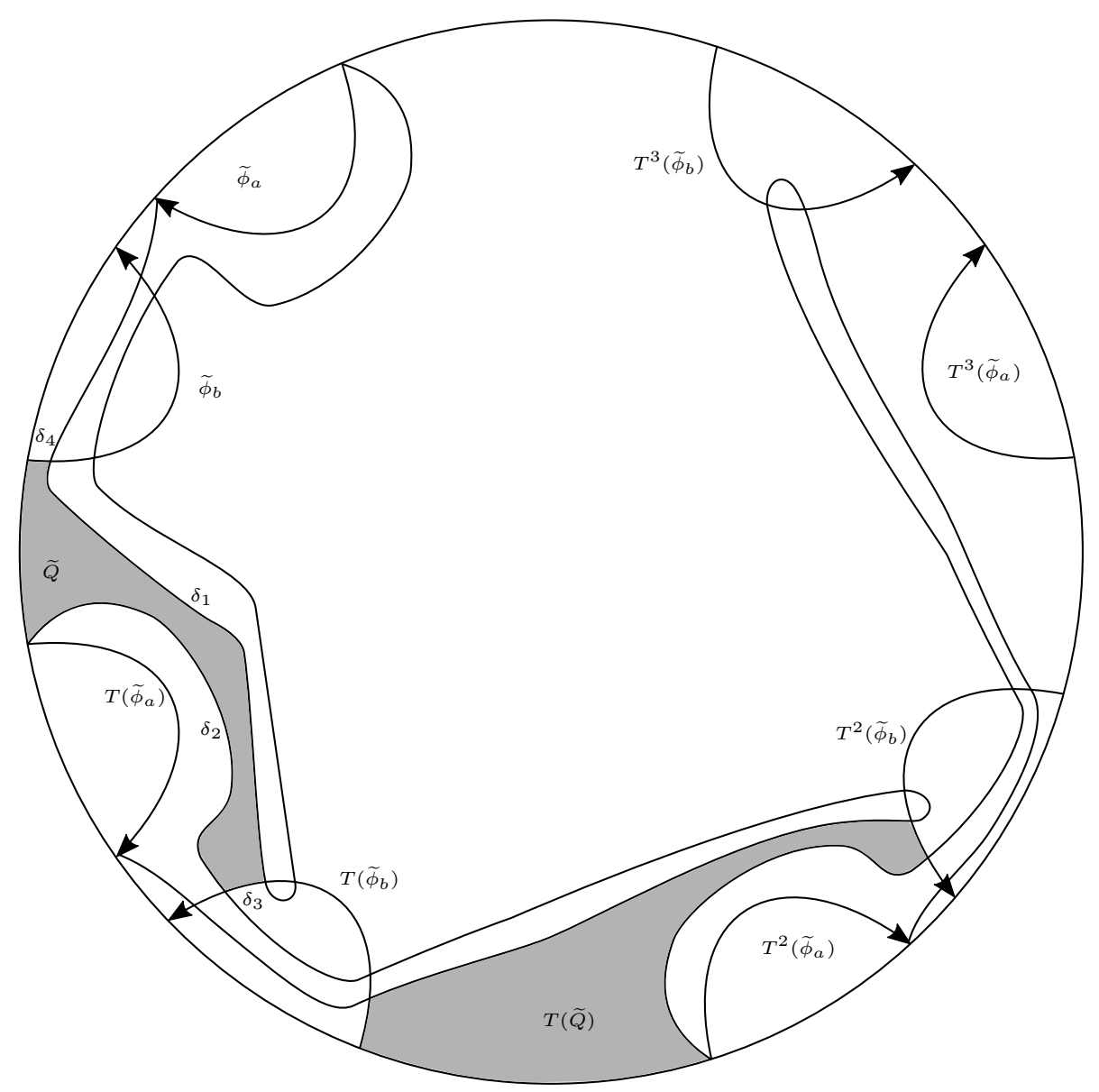

Figura 19: A intersecção de $\widetilde{Q}$ e $T(\widetilde{Q})$ é vazia.

Corolário 4.8. Se $k=1$ então $\widetilde{Q}$ é homeomorfo à sua projeção em $\widehat{\operatorname{dom}}(I)$.

Corolário 4.9. Se $k=1$ então $\delta_{1}, \delta_{2}, \delta_{3}$ e $\delta_{4}$ são homeomorfos às suas projeções em $\widehat{\operatorname{dom}}(I)$.

Por mais que o corolário 4.9 esteja implícito no corolário 4.8, é importante e extremamente válido ressaltar este, pois a fronteira do conjunto $\widetilde{Q}$ será de grande valia a este trabalho. 
Quando $k>1$, é possível que aconteça de $\delta_{3}$ intersectar $T\left(\delta_{1}\right)$ e assim, a intersecção de $\widetilde{Q} \operatorname{com} T(\widetilde{Q})$ é não vazia. Caso esta intersecção seja vazia para $k>1$, é possível mostrar, da mesma forma feita com $k=1$, que a entropia de $f$ é ao menos $\log (2 k) / n$, e, portanto, isto explica o porque da conjectura 4.2. De agora em diante, a menos de menção em contrário, iremos supor que $k=1$.

Considere novamente o espaço de recobrimento anular $\widehat{\operatorname{dom}}(I)=\widetilde{\operatorname{dom}}(I) / T$ e denote $\pi_{1}: \widetilde{\operatorname{dom}}(I) \rightarrow \widehat{\operatorname{dom}}(I)$ a aplicação projeção no espaço de recobrimento anular e $\widehat{f}=\pi_{1} \circ \widetilde{f}$. Se $\phi_{\widehat{\gamma}(a)}$ e $\phi_{\widehat{\gamma}(b)}$ denotam a projeção de $\phi_{\widetilde{\gamma}(a)}$ e $\phi_{\widetilde{\gamma}(b)}$ em $\widehat{\operatorname{dom}}(I)$ respectivamente, sabemos pela demonstração do lema 4.3, que ambos caminhos são homoclínicos ao mesmo fim. Denotaremos este fim por $N$ e o outro fim por $S$. Pelo corolário 4.8, $\widehat{Q}$, a projeção de $\widetilde{Q}$ em $\widehat{\operatorname{dom}}(I)$, possui como fronteira as projeções por $\pi_{1}$ dos conjuntos $\delta_{1}, \delta_{2}, \delta_{3}$ e $\delta_{4}$. Pela construção de $\widetilde{Q}$, $\widehat{Q}$ é homoclínico à $N$ e existe uma vizinhança de $S$ que não intersecta $\widehat{Q}$.

Considere agora $\widehat{\widehat{\operatorname{dom}}}(I)=\widehat{\operatorname{dom}}(I) \cup\{N\} \cup\{S\}$ o seu fecho de $\widehat{\operatorname{dom}}(I)$ em uma esfera topológica. Denotaremos $i: \widehat{\operatorname{dom}}(I) \rightarrow \widehat{\operatorname{dom}}(I)$ a inclusão nesta esfera. Denotaremos também por $\overline{\widehat{f}}$ a extensão de $\widehat{f}$ a $\widehat{\widehat{\operatorname{dom}}}(I)$ que fixa $\{N\}$ e $\{S\}, \overline{\widehat{\gamma}}=i \circ \widetilde{\gamma}, \widehat{\delta}_{i}=\left(i \circ \pi_{1}\right)\left(\delta_{i}\right) \cup\{N\}$ para $i=2$ e $4,{\overline{\delta_{i}}}_{i}=\left(i \circ \pi_{1}\right)\left(\delta_{i}\right)$ para $i=1$ e 3 e por $\overline{\widehat{Q}}=\left(i \circ \pi_{1}\right)(\widetilde{Q}) \cup\{N\}$.

Vamos mostrar que $\widehat{\widehat{Q}}$ é uma ferradura topológica para $(\overline{\widehat{f}})^{-1}$, ou seja, existe um subconjunto $\widehat{\widehat{Z}}$ invariante por $(\overline{\widehat{f}})^{-1}$, tal que sua restrição a $\widehat{\widehat{Z}}$ é uma extensão do shift $\sigma: \Sigma_{2} \rightarrow \Sigma_{2}$.

Observe que $\widehat{\widehat{Q}}$ tem como fronteira os conjuntos $\overline{\widehat{\delta}}_{j}$, para $j=1,2,3,4$. Para cada $i=0,1$, considere

$$
\widetilde{Q}_{i}=\widetilde{f}^{-1}(\widetilde{Q}) \cap T^{-i}(\widetilde{Q})
$$

Posteriormente vamos mostrar que esses conjuntos são não vazios. Estes conjutos são importantes pois através deles determinaremos as "pernas da ferradura".

Observação 4.10. $\widetilde{Q}_{0} \cap \widetilde{Q}_{1}=T\left(\widetilde{Q}_{0}\right) \cap \widetilde{Q}_{1}=\widetilde{Q}_{0} \cap T\left(\widetilde{Q}_{1}\right)=\emptyset$. Isso se deve ao fato de que $\widetilde{Q} \cap T \widetilde{Q}=\emptyset$ e que, pelo lema dos discos livres (lema 2.4), temos então que $\widetilde{Q} \cap T^{i}(\widetilde{Q})=\emptyset, \forall i \neq 0$. Para cada $i=0,1$, as componentes conexas de $\widetilde{Q}_{i}$ são duas a duas disjuntas módulo $T$, ou seja, se $\widetilde{Q}_{i}^{1}$ e $\widetilde{Q}_{i}^{2}$ são componentes conexas distintas de $\widetilde{Q}_{i}$, então $\widetilde{Q}_{i}^{1} \cap T \widetilde{Q}_{i}^{2}=\emptyset$. 
Para demonstrar o teorema principal deste trabalho (Teorema 4.1), é necessário a seguinte proposição:

Proposição 4.11. Seja $\overline{\widehat{K}}$ um contínuo em $\overline{\widehat{Q}}$ que une $\overline{\widehat{\delta}_{1}}$ a $\overline{\widehat{\delta}_{2}}$. Então existem pelo menos 2 subcontínuos de $\widehat{\widehat{K}}$, disjuntos, que quando aplicamos $(\overline{\widehat{f}})^{-1}$ unem $\widehat{\widehat{\delta}}_{1}$ a $\widehat{\widehat{\delta}}_{2}$ e estão contidos em $\overline{\widehat{Q}}$.

A demostração da proposição acima se dará com a demonstração de alguns lemas.

Lema 4.12. $\widetilde{f}^{-1}(\widetilde{Q}) \cap\left(T\left(\widetilde{\phi}_{b}\right) \cup\left(\widetilde{\phi}_{b}\right)\right)=\emptyset$.

Demonstração. Observe que $\widetilde{Q}$ está contido em $R\left(T\left(\widetilde{\phi}_{b}\right) \cup\left(\widetilde{\phi}_{b}\right)\right)$. Como $T\left(\widetilde{\phi}_{b}\right)$ e $\widetilde{\phi}_{b}$ são linhas de brouwer o resultado é imediato.

Aplicando $\widetilde{f}^{-1}$ a $\widetilde{Q}$, temos que $\widetilde{f}^{-1}\left(\delta_{1}\right) \subseteq \widetilde{\phi}_{a}$ e $\widetilde{f}^{-1}\left(\delta_{2}\right) \subseteq T \widetilde{\phi}_{a}$. Além disso, $\widetilde{f}^{-1}(\widetilde{Q}) \cap \overline{L\left(\widetilde{\phi}_{b}\right)}=\emptyset$ e $\widetilde{f}^{-1}(\widetilde{Q}) \cap T\left(\widetilde{\phi}_{b}\right)=\emptyset$. Isto significa que a imagem de $\widetilde{Q}$ por $f^{-1}$ é um conjunto que encontra $\widetilde{\phi}_{a}$ e $T\left(\widetilde{\phi}_{a}\right)$ mas não encontra o fecho da esquerda de $\widetilde{\phi}_{b}$ nem de $T\left(\widetilde{\phi}_{b}\right)$.

Primeiramente, iremos provar a Proposição 4.11 para o caso em que o contínuo não contenha o ponto $N$.

Lema 4.13. Seja $\overline{\widehat{K}}$ um contínuo em $\overline{\widehat{Q}}$ que une $\overline{\widehat{\delta}_{1}}$ a $\overline{\widehat{\delta}_{2}}$ e tal que $N \notin \overline{\widehat{K}}$. Então existem pelo menos 2 subcontinuos de $\widehat{\widehat{K}}$, disjuntos, que quando aplicamos $(\overline{\widehat{f}})^{-1}$ unem $\overline{\widehat{\delta}_{1}}$ a $\widehat{\widehat{\delta}}_{2}$ e estão contidos em $\overline{\widehat{Q}}$.

Demonstração. Seja $\overline{\widehat{K}}$ um contínuo como na hipótese e seja $\widetilde{K}=\pi_{1}^{-1} i^{-1}(\overline{\widehat{K}}) \cap \widetilde{Q}$. Segue que $\widetilde{K}$ é homeomorfo a $\overline{\widehat{K}}$ e é compacto, pois $\overline{\widehat{K}}$ não contém o ponto $N$.

Como $\widetilde{\phi}_{a}$ e $T \widetilde{\phi}_{a}$ estão em componentes conexas distintas do complementar de $L\left(T^{-1}\left(\widetilde{\phi}_{b}\right)\right) \cup T^{-1}(\widetilde{Q}) \cup L\left(\widetilde{\phi}_{b}\right)$ e $\widetilde{f}^{-1}(\widetilde{K})$ encontra $\widetilde{\phi}_{a}$ e $T \widetilde{\phi}_{a}$ e não encontra $L\left(T^{-1}\left(\widetilde{\phi}_{b}\right)\right) \cup L\left(\widetilde{\phi}_{b}\right)$, segue que existe um subcontínuo $\widetilde{K}_{1}$ de $\widetilde{K}$ tal que $\widetilde{f}^{-1}\left(\widetilde{K}_{1}\right)$ está contido em $T^{-1}(\widetilde{Q})$ e encontra $T^{-1} \delta_{1}$ e $T^{-1} \delta_{2}$.

Um argumento semelhante mostra que existe um subcontínuo $\widetilde{K}_{0}$ de $\widetilde{K}$ tal que $\widetilde{f}^{-1}\left(\widetilde{K}_{0}\right)$ está contido em $\widetilde{Q}$ e encontra $\delta_{1}$ e $\delta_{2}$ (veja figura (20)). Observe que aqui estamos mostrando que $\widetilde{Q}_{i} \neq \emptyset$ para $i=0,1$, pois $\widetilde{K}_{i}$ está contido em $\widetilde{Q}_{i}$, para $i=0,1$. 


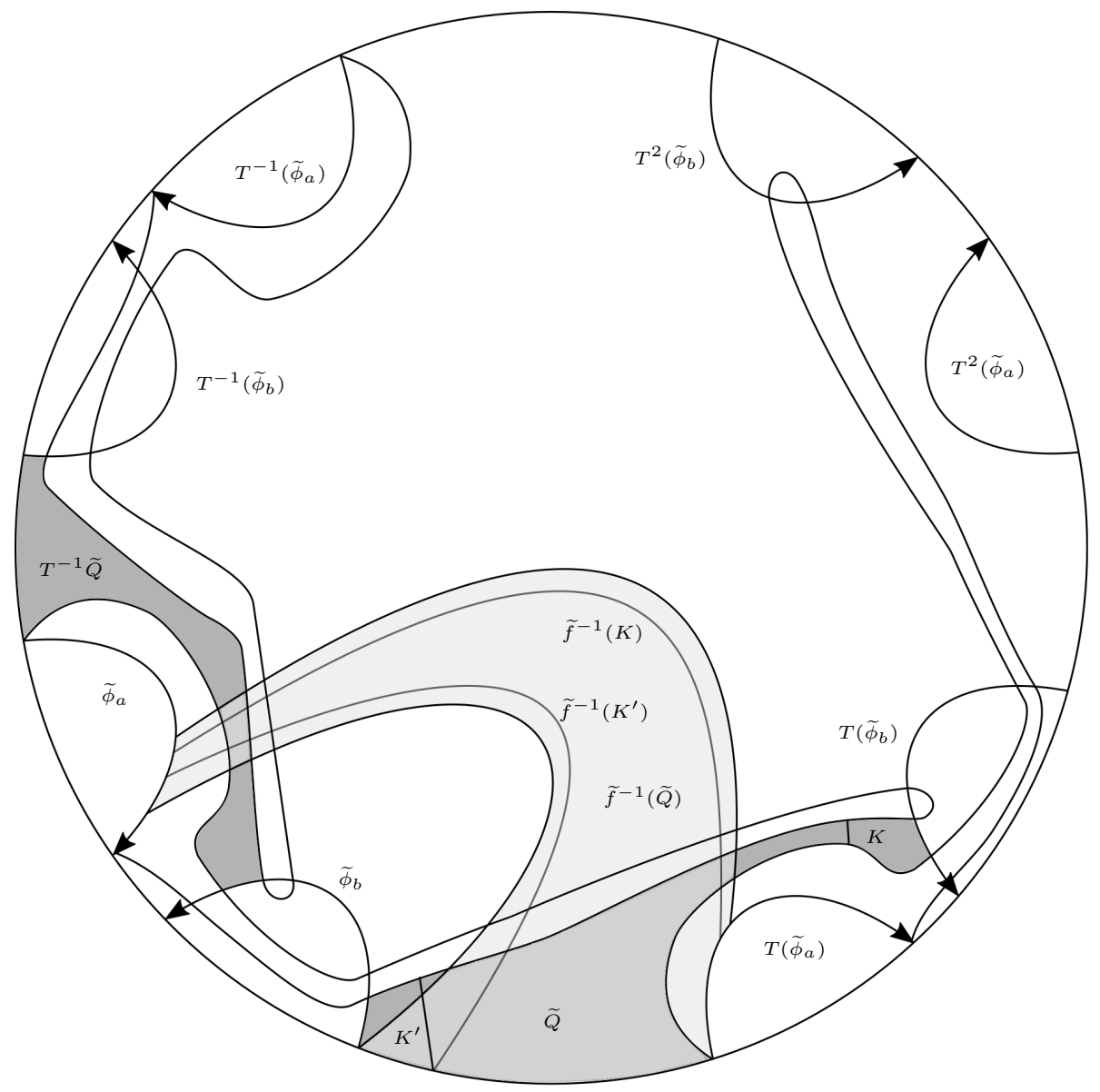

Figura 20: Existência de $\widetilde{K}_{0}$ e $\widetilde{K}_{1}$.

Assim, temos 2 subcontínuos de $\widetilde{K}$ disjuntos (pela observação 4.10) tal que para cada $j=0,1, \pi_{1}\left(\widetilde{f}^{-1}\left(\widetilde{K}_{j}\right)\right)$ encontra $\widehat{\delta}_{1}$ e $\widehat{\delta}_{2}$, está contido em $\widehat{Q}$.

Portanto, para cada $j=0,1$, temos que $\widehat{\widehat{K}}_{j}=i\left(\pi_{1}\left(\widetilde{K}_{j}\right)\right)$ é um subconjunto de $\overline{\widehat{K}}$ tal que $(\overline{\widehat{f}})^{-1}\left(\widehat{\widehat{K}}_{j}\right)$ encontra $\overline{\widehat{\delta}_{1}}$ e $\widehat{\widehat{\delta}}_{2}$ e está contido em $\overline{\widehat{Q}}$. Além disso, $\widehat{\widehat{K}}_{1} \cap \widehat{\widehat{K}}_{0}=\emptyset$, ou seja, temos 2 subcontínuos com essa propriedade e isto encerra a demonstração.

Lema 4.14. Seja $\overline{\widehat{K}}$ um continuo em $\overline{\widehat{Q}}$ que une ${\widehat{\widehat{\delta}_{1}}}_{\text {a }}$ a $\widehat{\widehat{\delta}}_{2}$ e tal que $N \in \overline{\widehat{K}}$. Então existem pelo menos 2 subcontinuos de $\widehat{\widehat{K}}$, disjuntos, que quando aplicamos $(\widehat{\widehat{f}})^{-1}$ unem $\overline{\widehat{\delta}_{1}}$ a $\overline{\widehat{\delta}_{2}}$ e estão contidos em $\overline{\widehat{Q}}$.

Demonstração. Seja $\widehat{\widehat{K}}$ como no enunciado. Considere $\widetilde{K}=\left[\pi_{1}^{-1} i^{-1}(\widehat{\widehat{K}} \backslash N)\right] \cap \widetilde{Q}$. $\widetilde{K}$ é um subconjunto de $\widetilde{Q}$ ilimitado. A mesma demonstração de antes nos 
mostra que é possível encontrar 2 subconjuntos de $\widetilde{K}$, denotados por $\widetilde{K}_{0}$ e $\widetilde{K}_{1}$, com apenas 1 deles ilimitado que vamos supor ser o $\widetilde{K}_{0}$ que está inteiramente contido em $\widetilde{Q}$ e encontra $\delta_{1}$. Além disso, o conjunto $\widetilde{K}_{1}$ é compacto e conexo e tal que $\widetilde{f}^{-1}\left(\widetilde{K}_{1}\right)$ encontra $T^{-1}\left(\widetilde{\delta}_{1}\right)$ e $T^{-1}\left(\widetilde{\delta}_{2}\right)$, e está contido em $T^{-1}(\widetilde{Q})$ (veja figura (20)).

Observe que $\widehat{\widehat{K}}_{1}=\left(i \pi_{1} \widetilde{K}_{1}\right) \cup\{N\}$ é um subconjunto de $\overline{\widehat{K}}$ e que $(\overline{\widehat{f}})^{-1}\left(\widehat{\widehat{K}}_{1}\right)$ encontra $\bar{\delta}_{1}$ e $\bar{\delta}_{2}$ (pois $N \in \bar{\delta}_{2}$ ) e está contido em $\overline{\widehat{Q}}$.

Portanto, qualquer contínuo $\overline{\widehat{K}}$ contido em $\overline{\widehat{Q}}$ que encontra $\overline{\widehat{\delta}}_{1}$ e $\overline{\widehat{\delta}}_{2}$, possui 2 subcontínuos disjuntos tal que a imagem por $(\overline{\widehat{f}})^{-1}$ está contida em $\widehat{\widehat{Q}}$ e encontra $\widehat{\widehat{\delta}}_{1}$ e $\overline{\widehat{\delta}}_{2}$.

Demonstração da Proposiçãa 4.11. Considere $\overline{\widehat{K}}$ um contínuo que que une $\overline{\widehat{\delta}}_{1}$ a $\overline{\widehat{\delta}_{2}}$. Caso $N$ não percença a $\overline{\widehat{K}}$, o lema 4.13 nos dá 2 subcontínuos de $\overline{\widehat{K}}$ disjuntos que quando aplicamos $(\overline{\widehat{f}})^{-1}$ encontram $\overline{\widehat{\delta}_{1}}$ e $\overline{\widehat{\delta}_{2}}$. Caso $N$ não pertença a $\overline{\widehat{K}}$, o lema 4.14 nos dá um resultado análogo. Portanto, em qualquer caso, temos 2 subconjuntos com a propriedade desejada, o que encerra a proposição.

Proposição 4.15. A entropia topológica de $\overline{\widehat{f}}$ é ao menos $\log (2)$.

Demonstração. Para provar este resultado faremos o uso da teoria de Kennedy e Yorke $([10])$ que foi explicada nas sessões anteriores. Defina end $_{0}$ e end $_{1}$ como sendo os conjuntos $\bar{\delta}_{1}$ e $\widehat{\delta}_{2}$ respectivamente. Se $K$ é uma conexão, então, por definição, $K$ é um contínuo que encontra end $d_{0}$ e end $d_{1}$. Pela proposição 4.11, temos que existem pelo menos 2 preconexões disjuntas, o que implica que o número de cruzamentos é $M=2$. Pelo teorema 1.3 , temos que $\bar{Q}$ é uma ferradura topológica de 2 pernas em $\widehat{\widehat{\operatorname{dom}}}(I)$. Assim, existe um subconjunto compacto $\overline{\widehat{Z}} \subseteq \overline{\widehat{Q}}$ invariante por $(\overline{\widehat{f}})^{-1}$ tal que sua restrição a $\overline{\widehat{Z}}$ é uma extensão do shift de Bernoulli $\sigma: \Sigma_{2} \rightarrow \Sigma_{2}$. Por conseguinte, a entropia de $(\overline{\widehat{f}})^{-1}$ é ao menos $\log 2$, e o mesmo vale para $\overline{\widehat{f}}$.

Considere para $m \geq 2$,

$$
\Sigma_{2}^{m}=\left\{a \in \Sigma_{2}: \forall j \in \mathbb{Z}, \exists i=i(j) \in\{0,1\} \text { tal que } a_{j+i} \neq 0\right\}
$$

$\Sigma_{2}^{m}$ é um subconjunto de $\Sigma_{2}$ tal que todo elemento não possui uma sequência com $m$ zeros seguidos ou mais. Considere $u: \bar{Z} \rightarrow \Sigma_{2}$ a função fator. Afirmação: 
$-u(N) \notin \Sigma_{2}^{m}$.

- $\sum_{2}^{m}$ é compacto.

A primeira é óbvia, e a segunda pode ser demonstrada pelo fato de $\Sigma_{2}^{m}$ ser fechado. Antes de provar o teorema principal desta tese, precisamos de um lema técnico:

Proposição 4.16. $h\left(\left.\sigma\right|_{\Sigma_{2}^{m}}\right) \rightarrow \log (2)$ quando $m \rightarrow \infty, m$ par.

Demonstração. Fixe $m \geq 2, m$ par. Vamos denotar $g_{m}=\left.\sigma\right|_{\Sigma_{2}^{m}}$. Observe que $\sigma$ é expansiva, logo vale que

$$
h\left(g_{m}\right) \geq \limsup _{s} \frac{\log \# \operatorname{Fix}\left(g_{m}^{s}\right)}{s} .
$$

Vamos usar que

$$
\limsup _{s} \frac{\log \# \operatorname{Fix}\left(g_{m}^{s}\right)}{s} \geq \limsup _{s} \frac{\log \# \operatorname{Fix}\left(g_{m}^{s m}\right)}{s m} .
$$

Daí, como

$$
\# \operatorname{Fix}\left(g_{m}^{s m}\right) \geq\left(\left(2^{m / 2}-1\right)\left(2^{m / 2}-1\right)\right)^{s}=\left(2^{m}-2(2)^{m / 2}+1\right)^{s},
$$

segue que

$$
\limsup _{s} \frac{\log \# \operatorname{Fix}\left(g_{m}^{s m}\right)}{s m} \geq \frac{\log \left(2^{m}-2(2)^{m / 2}+1\right)}{m}
$$

Assim,

$$
h\left(g_{m}\right) \geq \frac{\log \left(2^{m}-2(2)^{m / 2}+1\right)}{m},
$$

para todo $m \geq 2, m$ par. Como

$$
\frac{\log \left(2^{m}-2(2)^{m / 2}+1\right)}{m} \rightarrow \log (2),
$$

quando $m \rightarrow \infty$, obtemos finalmente que

$$
\lim _{m} h\left(g_{m}\right) \geq \log (2)
$$


Como $h\left(g_{m}\right) \leq \log (2)$, para todo $m$, o resultado segue.

Demonstração do Teorema 4.1. Considere $u: \bar{Z} \rightarrow \Sigma_{2}$ a função fator. Suponha primeiro que $N \notin \overline{\widehat{Z}}$. Como $S \notin \overline{\widehat{Q}}$, segue que $S \notin \widehat{\widehat{Z}}_{n}$. Denote $\widehat{Z}=i^{-1}(\widehat{\widehat{Z}})$ e $Z=\widehat{\pi}(\widehat{Z})$ (Veja figura 21.).

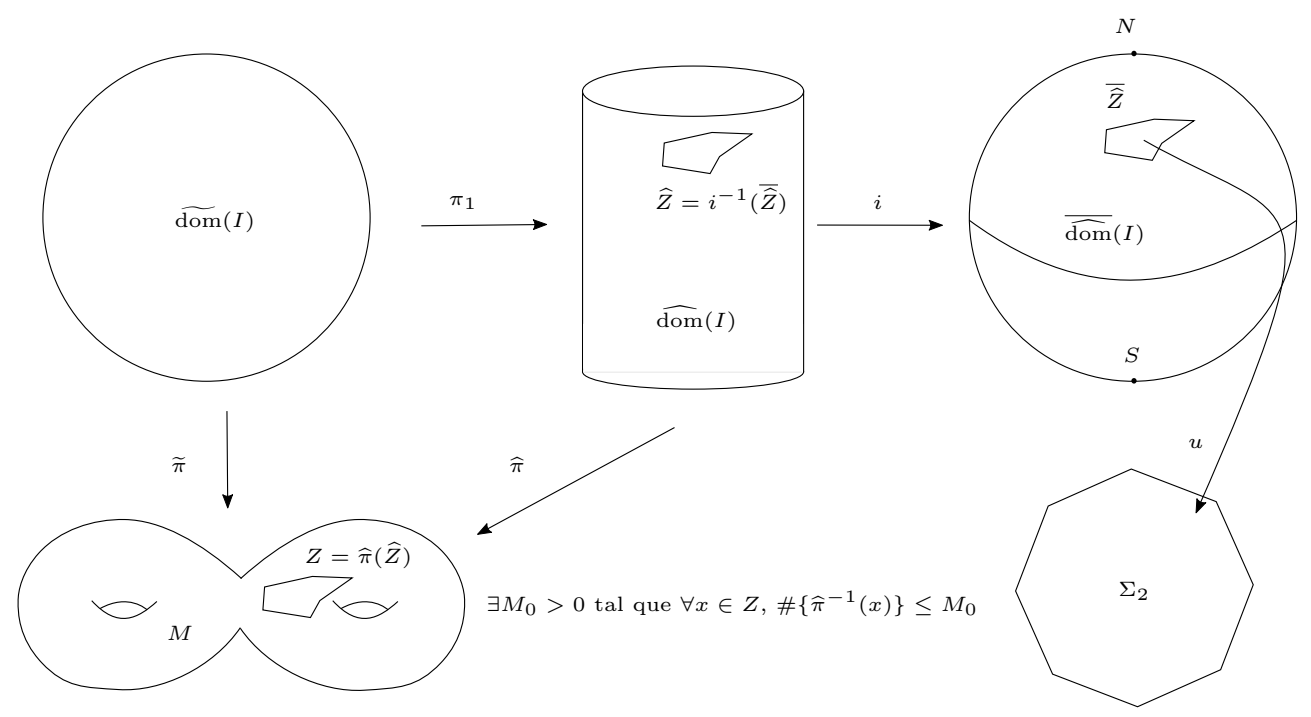

Figura 21: $N \notin \overline{\widehat{Z}}$.

Existe $M_{0}>0$ tal que $\forall x \in Z, \#\left\{\widehat{\pi}^{-1}(x)\right\} \leq M_{0}$. Isso decorre do fato que $i^{-1}(\overline{\widehat{Z}})$ é compacto. Assim, se $h(f)$ denota a entropia de $f$, vale que:

$$
h(f) \geq h\left(\left.f\right|_{Z}\right)=h\left(\left.\widehat{f}\right|_{\widehat{Z}}\right) \geq h(\sigma)=\log (2) .
$$

Suponha agora que $N \in \overline{\widehat{Z}}$. Como $N$ é um ponto fixo de $(\overline{\widehat{f}})^{-1}$, segue que $u(N)$ só pode ser uma sequência fixa de $\sigma$. Vamos supor, sem perda de generalidade, que $u(N)$ é a sequência de zeros. Pelo fato que $u(N) \notin \Sigma_{2}^{m}$ e que $\Sigma_{2}^{m}$ é compacto segue que $\widehat{\widehat{Z}}_{m}=u^{-1}\left(\Sigma_{2}^{m}\right)$ é um subconjunto compacto de $\widehat{\widehat{\operatorname{dom}}}(I)$ tal que $N \notin \widehat{\widehat{Z}}_{m}$. Além disso, como $S \notin \widehat{Q}$, segue que $S \notin \widehat{Z}_{m}$.

Observe que $\widehat{Z}_{m}$ é $(\widehat{f})^{-1}$ invariante e que a restrição de $u$ a $\widehat{Z}_{m}$ é uma semiconjugação entre $\left.(\widehat{\widehat{f}})^{-1}\right|_{\widehat{Z}_{m}}$ e $\left.\sigma\right|_{\Sigma_{2}^{m}}$. Assim, se $h(f)$ denota a entropia de $f$, temos que

$$
h\left(\left.(\overline{\widehat{f}})^{-1}\right|_{\overline{\bar{Z}}_{m}}\right) \geq h\left(\left.\sigma\right|_{\Sigma_{2}^{m}}\right) .
$$


Como $\{N, S\} \cap \widehat{Z}_{m}=\emptyset$, segue que $\left.i\right|_{i^{-1}\left(\bar{Z}_{m}\right)}: i^{-1}\left(\widehat{Z}_{m}\right) \rightarrow \widehat{Z}_{m}$ é um homeomorfismo. Vamos denotar $\widehat{Z}_{m}=i^{-1}\left(\widehat{Z}_{m}\right)$ Considere $\hat{\pi}: \widehat{\operatorname{dom}}(I) \rightarrow \operatorname{dom}(I)$ a projeção e seja $Z_{m}=\widehat{\pi}\left(\widehat{Z}_{m}\right)$. Veja figura 22 .

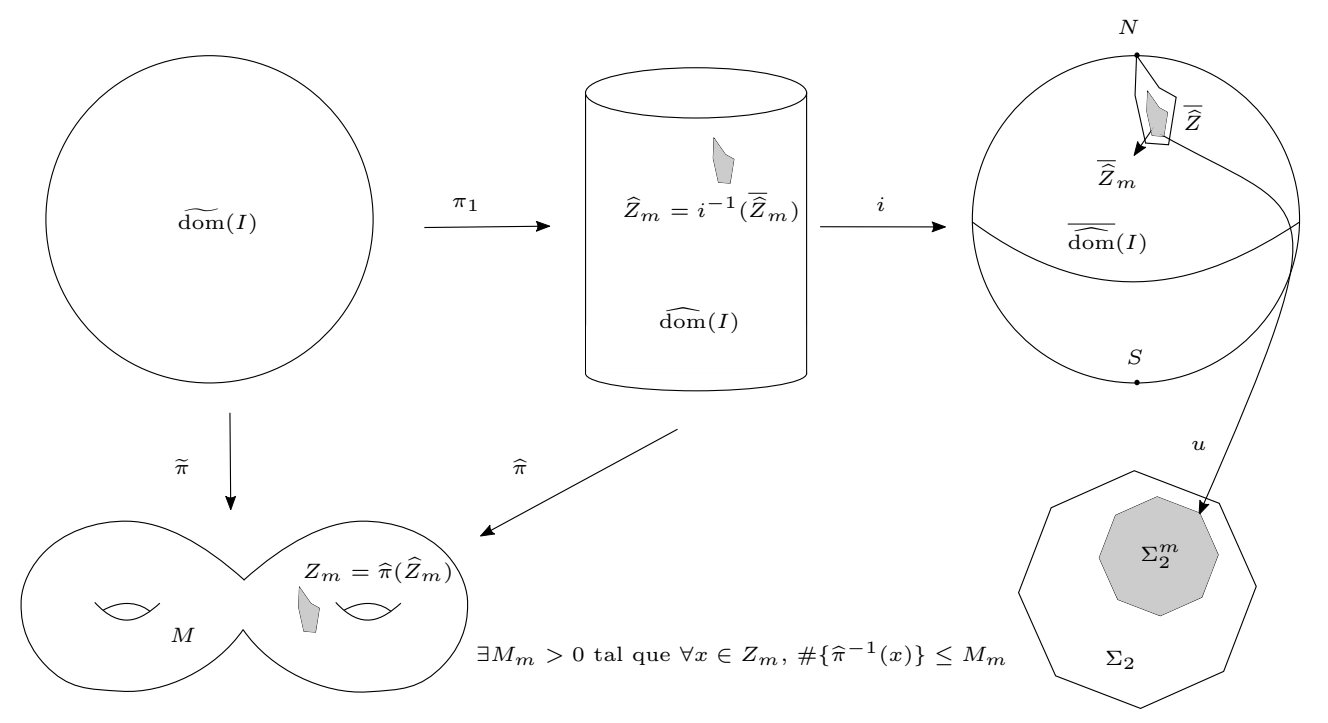

Figura 22: $N \in \overline{\widehat{Z}}$.

Como $\widehat{Z}_{m}$ é compacto, e portanto $\widehat{Z}_{m}$ é compacto, segue que $Z_{m}$ é um subconjunto compacto de $\operatorname{dom}(I)$ e todo ponto em $Z_{m}$ tem finitos levantamentos em $\widehat{Z}_{m}$ com uma limitação uniforme, ou seja, existe $M_{m}>0$ tal que $\forall x \in Z_{m}$, $\#\left\{\widehat{\pi}^{-1}(x)\right\} \leq M_{m}$. Assim vale:

$$
h(f)=h\left(f^{-1}\right) \geq h\left(\left.f^{-1}\right|_{Z_{m}}\right)=h\left(\left.(\widehat{f})^{-1}\right|_{\widehat{Z}_{m}}\right)=h\left(\left.(\widehat{\widehat{f}})^{-1}\right|_{\widehat{Z}_{m}}\right) \geq h\left(\left.\sigma\right|_{\Sigma_{2}^{m}}\right) .
$$

Para concluir o teorma, basta ver que $h\left(\left.\sigma\right|_{\Sigma_{2}^{m}}\right)$ converge para $\log (2)$ quando $n \rightarrow \infty$. Na verdade, basta mostrar para qualquer sequência que vai para $o$ infinito, e, por isso, é suficiente mostrar para o caso $m$ par, e isto foi feito na Proposição 4.16: A desigualdade $h(f) \geq h\left(\left.\sigma\right|_{\Sigma_{2}^{m}}\right)$ vale para todo $m \geq 2$. Como $h\left(\left.\sigma\right|_{\Sigma_{2}^{m}}\right) \rightarrow \log (2)$ quando $m \rightarrow \infty, m$ par, segue que

$$
h(f) \geq \log (2) .
$$


A prova acima mostra que se $\gamma$ for 1-admissível então a entropia de $f$ é ao menos $\log (2)$. Se $\gamma$ for $n$-admissível, observe que a entropia de $f^{n}$ é ao menos $\log (2)$ e consequentemente, a entropia de $f$ é pelo menos $\log 2 / n$ e isto encerra o teorema principal.

\subsection{Aplicação}

Nessa seção vamos mostrar uma aplicação do resultado principal deste trabalho, que consiste em uma estimativa melhor de um resultado de Le Calvez e Tal em [16] que possui conexão com o estudo da mínima entropia de tranças puras em $\mathbb{S}^{2}$.

Um caminho $\gamma: \mathbb{R} \rightarrow M$ tal que $\gamma(t+1)=\gamma(t)$ para todo $t \in \mathbb{R}$ levanta uma aplicação contínua $\Gamma: \mathbb{T}^{1} \rightarrow M$, onde $\mathbb{T}^{1}=\mathbb{R} / \mathbb{Z}$. A aplicação $\Gamma$ é chamada de loop e $\gamma$ o seu levantamento natural. Se $n \geq 1$, $\Gamma^{n}$ denota o loop levantado pelo caminho $t \rightarrow \gamma(n t)$. Um loop $\Gamma: \mathbb{T}^{1} \rightarrow \operatorname{dom}(I)$ é chamado de positivamente transverso (ou simplesmente de transverso) para $\mathcal{F}$ se for o caso para seu levantamento natural $\gamma: \mathbb{R} \rightarrow \operatorname{dom}(I)$. Dois loops $\Gamma: \mathbb{T}^{1} \rightarrow \operatorname{dom}(I)$ e $\Gamma^{\prime}: \mathbb{T}^{1} \rightarrow \operatorname{dom}(I)$ são ditos equivalentes se existem dois levantamentos $\widetilde{\gamma}: \mathbb{R} \rightarrow \widetilde{\operatorname{dom}}(I)$ e $\widetilde{\gamma}^{\prime}: \mathbb{R} \rightarrow \widetilde{\operatorname{dom}}(I)$ de $\Gamma$ e $\Gamma^{\prime}$ respectivamente, uma transformação de recobrimento $T$ e um homeomorfismo que preserva a orientação $h: \mathbb{R} \rightarrow \mathbb{R}$, tal que para todo $t \in \mathbb{R}$, temos:

$$
\widetilde{\gamma}(t+1)=T(\widetilde{\gamma}(T)), \widetilde{\gamma}^{\prime}(t+1)=T\left(\widetilde{\gamma}^{\prime}(T)\right), h(t+1)=h(t)+1 \text { e } \phi_{\widetilde{\gamma}^{\prime}(h(t))}=\phi_{\widetilde{\gamma}(t)} \text {. }
$$

Um loop transverso $\Gamma$ é chamado de primo se não existe loop transverso $\Gamma^{\prime}$ e inteiro $n \geq 2$ tal que $\Gamma$ seja equivalente a $\Gamma^{\prime n}$. A demonstração das duas próximas proposições podem ser encontradas em [16], que são as proposições 2 e 7 respectivamente.

Proposição 4.17. Seja $\mathcal{F}$ uma folheação singular orientada em $\mathbb{S}^{2}$ e $\gamma: \mathbb{R} \rightarrow \mathbb{S}^{2}$ um caminho transverso fechado. São equivalentes:

- $\gamma$ não tem $\mathcal{F}$ autointersecção transversa. 
- existe um loop transverso simples $\Gamma^{\prime}$ tal que $\gamma$ é equivalente ao levantamento natural $\gamma^{\prime}$ de $\Gamma^{\prime}$.

Proposição 4.18. Seja $\mathcal{F}$ uma folheação singular orientada em uma superfície orientável $M$ e $\Gamma: \mathbb{T}^{1} \rightarrow M$ um loop transverso homólogo a zero em $M$ com uma $\mathcal{F}$ autointersecção transversa. Se $\gamma: \mathbb{R} \rightarrow M$ é o levantamento natural de $\Gamma$, então $\left.\gamma\right|_{[0,2]}$ tem uma $\mathcal{F}$ autointersecção transversa.

Assim, podemos enunciar e provar o principal resultado desta seção:

Teorema 4.19. Seja $f$ um homeomorfismo que preserva a orientação em $\mathbb{S}^{2} e$ I uma isotopia maximal. Assuma que existe um ponto $x \in \operatorname{dom}(I)$ fixo para $f$ tal que o loop naturalmente definido pela trajetória $I(x)$ não é homotópico em $\operatorname{dom}(I)$ a um múltiplo de um loop simples. Então a entropia de $f$ é pelo menos igual a $\log (2) / 2$.

Demonstração. Seja $\mathcal{F}$ uma folheação transversa para I. Pela hipótese do loop transverso $\Gamma$ associado a $x$ não é múltiplo de um loop simples, então pela proposição 4.17, segue que $\Gamma$ tem uma $\mathcal{F}$ autointersecção transversa. Se $\gamma$ é o levantamento natural de $\Gamma$, então, claramente pela definição de $\Gamma$, para todos os inteiros $N>0,\left.\gamma\right|_{[0, N]}$ é admissível de ordem $N$. Segue daí que pela proposição $4.18,\left.\gamma\right|_{[0,2]}$ tem uma $\mathcal{F}$ autointersecção transversa. Assim, $\left.\gamma\right|_{[0,2]}$ é um caminho transverso admissível de ordem 2 que possui uma $\mathcal{F}$ autointersecção transversa. O teorema segue diretamente do teorema 4.1.

Os pesquisadores Le Calvez e Tal provaram ([16]) que nas mesmas condições do Teorema 4.19, que a entropia de $f$ deve ser no mínimo $\log (2) / 4$. Aqui neste trabalho foi possível melhorar esta estimativa utilizando o Teorema 4.1.

Uma pergunta surge naturalmente do resultado acima:

- O resultado se mantém se trocarmos $\mathbb{S}^{2}$ por uma superfície orientável $M$ qualquer?

Esta questão acima ainda continua em aberto. A dificuldade de se replicar a demonstração acima para uma superfície $M$ qualquer é devido ao fato que não se tem até o momento uma prova de que, no caso geral, o caminho $\gamma$ possua uma autointersecção transversa. 


\section{Referências}

[1] Viana, M.; Oliveira, K.: Fundamentos da Teoria Ergódica, Sociedade Brasileira de Matemática, 2014.

[2] Adler, R.L.; Konheim, Allan G.; McAndrew, M.H.: Topological entropy, Trans. Am. Math. Soc. 114 (2), 309-319 (1965).

[3] Bowen, R.: Entropy for Group Endomorphisms and Homogeneous Spaces, Trans. Am. Math. Soc. 153, pp. 401-414 (1971).

[4] Katok, A.: Lyapounov exponents, entropy and periodic orbits for diffeomorphisms, Publications Mathématiques de l'I.H.É.S.51 (1980), no. 4, 131173.

[5] Rees, M.: A minimal positive entropy homeomorphism of the 2-torus, J. London Math. Soc. 23 (1981), 537-550.

[6] Béguin F.; Crovisier S.; Le Roux F.: Construction of curious minimal uniquely ergodic homeomorphisms on manifolds: the Denjoy-Rees technique, Ann. Sci. École Norm. Sup. (4) 40 (2007), no. 2, 251-308.

[7] Franks J.; Handel M.: Entropy zero area preserving diffeomorphisms of $\mathbb{S}^{2}$, Geom. Topol., 16(2013), no.4, 2187-2284.

[8] Smale, S..: Differentiable dynamical systems, Bull. Amer. Math. Soc.73(1967), 747-817.

[9] Burns, K.; Weiss, H.: A geometric criterion for positive topological entropy Math. Phys. 172 (1995), 95-118.

[10] Kennedy, J.; Yorke, J. A.: Topological horseshoes Trans. Amer. Math. Soc., v. 353, no. 6, p. 2513-2530, 2001.

[11] Franks, J.: A new proof of the Brouwer plane translation theorem, ETDS, 12, no. 2, 1992, 217-226. 
[12] Le Calvez, P.: Une version feuilletée du théorème de translation de Brouwer, Comment. Math. Helv., 21 (2004), 229-259.

[13] Le Calvez, P.: Une version feuilletée équivariante du théorème de translation de Brouwer, Publ. Math. Inst. Hautes Études Sci. (2005), no. 102, 1-98.

[14] Misiurewicz, M.; Zieman, K.: Rotation sets for maps of tori, J. London Math. Soc., 40 (1989), no. 3, 490-506.

[15] Epstein, D. B. A.: Curves on 2-manifolds and isotopies, Acta Math. 115 (1966), 83-107.

[16] Tal, F. A.; Le Calvez, P.: Forcing theory for transverse trajectories of surface homeomorphisms. Invent. Math., v.212, n.2, p. 619-729, 2018.

[17] Jaulent O.: Existence d'un feuilletage positivement transverse à un homéomorphisme de surface, Ann. Inst. Fourier (Grenoble), 64 (2014), no. 4, 1441-1476.

[18] Béguin, F.; Crovisier, S.; Le Roux, F.: Fixed point sets of isotopies on surfaces, arXiv:1610.00686 (2016).

[19] Tal, F. A.; Le Calvez, P.: Topological horseshoes for surface homeomorphisms, arXiv:1803.04557 (2018).

[20] de Carvalho, A.; Lyubich M.; Martens M.: Renormalization in the Hénon family, I: Universality but non-rigidity. J. Stat. Phys., 121 (2005), no. 5-6, 611-669.

[21] Lyubich M.; Martens M.: Renormalization in the Hénon family, II: the heteroclinic web. Invent. Math., 186 (2011), no. 1, 115-189.

[22] Hazard, P.; Martens, M.; Tresser, C.: Infinitely many moduli of stability at the dissipative boundary of chaos. To appear in Trans. Amer. Math. Soc. (2017). 